\title{
Syntactic predictions and asyntactic comprehension in aphasia: Evidence from scope relations
}

\author{
Maria Varkanitsa $^{\text {1a }}$, Dimitrios Kasselimis ${ }^{\text {b,c }}$, Andrew J.B. Fugard ${ }^{\text {d }}$, Ioannis \\ Evdokimidis $^{\mathrm{c}}$, Judit Druks ${ }^{\mathrm{a}}$, Constantin Potagas ${ }^{\mathrm{c}}$, Hans Van de Koot ${ }^{\mathrm{a}}$ \\ ${ }^{a}$ Department of Linguistics, University College London, UK \\ ${ }^{b}$ Department of Psychology, University of Crete, Greece \\ ${ }^{c}$ Department of Neurology, National \& Kapodistrian University of Athens, Greece \\ ${ }^{\mathrm{d} D e p a r t m e n t ~ o f ~ C l i n i c a l, ~ E d u c a t i o n a l ~ \& ~ H e a l t h ~ P s y c h o l o g y, ~ U n i v e r s i t y ~ C o l l e g e ~}$ \\ London, UK
}

\section{Corresponding author}

Maria Varkanitsa

Room 102, Chandler House

2 Wakefield Street, London WC1N 1PF

$+447526168197$

maria.varkanitsa.09@ucl.ac.uk

\begin{abstract}
People with aphasia (PWA) often fail to understand syntactically complex sentences. This phenomenon has been described as asyntactic comprehension and has been explored in various studies cross-linguistically in the past decades. However, until now there has been no consensus among researchers as to the nature of sentence comprehension failures in aphasia. Impaired representations accounts ascribe comprehension deficits to loss of syntactic knowledge, whereas processing/resource reduction accounts assume that PWA are unable to use syntactic knowledge in comprehension due to resource limitation resulting from the brain damage. The aim of this paper is to use independently motivated psycholinguistic models of sentence processing to test a variant of the processing/ resource reduction accounts that we dub the Complexity Threshold Hypothesis. According to this hypothesis, PWA are capable of building well-formed syntactic representations, but, because their resources for language processing are limited, their syntactic parser fails when processing complexity exceeds a certain threshold. The source of complexity investigated in the experiments reported in this paper is syntactic prediction. We conducted two experiments involving comprehension of sentences with different types of syntactic dependencies, namely dependencies that do not require syntactic prediction (i.e. unpredictable dependencies in sentences that require Quantifier Raising) and

\footnotetext{
${ }^{1}$ Present address

Neuropsychology Laboratory

Massachusetts General Hospital - Harvard Medical School

100 Cambridge Street \#2000

Boston, MA 02114
}

Tel: 617.505.7693
\end{abstract}


dependencies whose resolution requires syntactic predictions at an early stage of processing based on syntactic cues (i.e. predictable dependencies in movementderived sentences). In line with the predictions of the Complexity Threshold Hypothesis, the results show that the agrammatic patients that participated in this study had no difficulties comprehending sentences with the former type of dependencies, whereas their comprehension of sentences with the latter type of dependencies was impaired.

Keywords: Aphasia, sentence comprehension deficits, syntactic predictions, contrastive focus, scope ambiguity

\section{Introduction}

Syntactic comprehension deficits in people with aphasia (PWA) and more specifically agrammatism have been the focus of language breakdown research crosslinguistically. Numerous studies have shown that PWA often experience difficulties in processing syntactically complex sentences. The main finding is that PWA are unable to construct a complete grammatical structure for an input sentence and identify the thematic role of the participants in the event described in the sentence. On the basis of this evidence, two types of accounts have been put forward to explain sentence comprehension deficits in aphasia, namely impaired representations accounts and processing/resource reduction accounts, which are briefly presented in the following paragraph.

Impaired representations accounts ascribe the deficit to loss of syntactic knowledge; they assume that patients suffer from a breakdown in their knowledge of grammar and therefore universal failure to apply specific operations (e.g. Trace Deletion Hypothesis; Grodzinsky, 1995). As a result, PWA's sentence comprehension relies on extralinguistic heuristic strategies. Therefore, under these accounts there are qualitative differences in processing routines between PWA and unimpaired adults due to loss of syntactic knowledge. Processing/resource reduction accounts, on the other hand, assume that the underlying grammatical knowledge is preserved, but PWA often fail to use it due to the brain damage that has limited the resources available for language processing. In the literature, there are several formulations of processing accounts. For instance, it has been suggested that, due to the resource reduction, PWA cannot carry out syntactic computations at the normal rate (slowed processing accounts; Friederici \& Kilborn, 1989; Kolk, 1995; Piñango, 2002). A number of suggestions have been made regarding the disturbances in cognitive processes that could underlie this slowdown, including slow activation or too-fast decay of structural information (Haarmann \& Kolk, 1991), slower automatic lexical (re)activation (Ferrill, Love, Walenski, \& Shapiro, 2012; Love, Swinney, \& Zurif, 2001), slowed online assembly of phrase structure (Burkhardt, Piñango, \& Wong, 2003), or delayed formation of the syntactic representation (Piñango, 2002).

Nevertheless, researchers agree that this slowdown allows non-syntactic mechanisms, otherwise suppressed in normal processing, to emerge, creating competition between the two possible sources of interpretation: the regular but slowed down syntactic 
mechanism and one that bypasses syntax altogether. Other researchers have suggested that resource reduction results in weakened (not slow downed) syntactic computations (weakened syntax; Avrutin, 2006; Dickey, Choy, \& Thompson, 2007; Dickey \& Thompson, 2009; Meyer, Mack, \& Thompson, 2012; Cynthia K Thompson \& Choy, 2009). Under this view, the outcome of syntactic computations is weakened and therefore vulnerable to influence from non-syntactic means for encoding information.

Of particular interest for the hypothesis presented in this paper are intermittent deficiency accounts. Similar to other processing accounts, intermittent deficiency accounts suggest that PWA suffer from resource limitations that result in parsing failures. However, they further suggest that those parsing failures are not constant. Rather, according to these accounts, resource limitations cause intermittent parsing failures. In those cases, PWA rely on non-syntactic alternative sources to assign an interpretation to the input sentence. Intermittent deficiency accounts have been supported by recent online studies which show that PWA sometimes manage to compute structures and meaning and that, when they do so, their processing routines resemble those of unimpaired speakers. Sometimes, however, their syntactic parser fails, with more frequent failures occurring when processing load is high or, in other words, complexity exceeds a certain threshold (Caplan, Waters, DeDe, Michaud, \& Reddy, 2007; Hanne, Sekerina, Vasishth, Burchert, \& De Bleser, 2011).

But, when is processing load considered too high for PWA? Although processing of syntactically complex sentences has been extensively investigated in the aphasia literature, the factors that contribute to complexity have rarely been discussed. Caplan and colleagues (2007) briefly discussed relativization and passivization as processes that increase complexity. Specifically, both online and end-of-sentence data from the self-paced listening task they administered showed that object relativization was associated with increased processing load (as reflected in lower accuracy and slower listening times) compared to subject relativization. Increased load was also found to be associated with passive compared to active sentences, as measured with listening times at the verb position. The authors concluded that different sentence types are not equally difficult to process but, rather, structural parameters in fact may affect performance. In their experimental manipulations, Hanne and colleagues (2011) associated high complexity with the canonicity of word order; their main finding was that comprehension of non-canonical sentences was severely impaired.

The aim of this paper is to combine aphasia-specific accounts with independently motivated computational models of sentence processing to allow the investigation of more fine-grained hypotheses regarding comprehension failures in PWA. Like many previous researchers we assume that PWA are capable of normal sentence processing but have resource limitations that cause their syntactic parser to suffer occasional intermittent failures in environments that push syntactic complexity above a certain threshold. For the purposes of the present study, we dub this hypothesis the Complexity Threshold Hypothesis. This view of comprehension failure in aphasia can be refined by combining it with what is known about processing complexity in the unimpaired population. Detailed psycholinguistic work has identified several factors that determine complexity in sentence processing and 
prominent among these are the intertwined effects of syntactic prediction and locality (see Dependency Locality Theory: Gibson, 1998, 2000; Grodner \& Gibson, 2005), similarity-based interference (see Cue-Based Retrieval Theory: Lewis \& Vasishth, 2005) and frequency (see Surprisal Theory: Levy, 2008). The present study makes use of these psycholinguistic results and focuses on the effects of syntactic prediction in sentence processing in PWA.

Syntactic predictions have played a major role in the study of sentence comprehension in the field of psycholinguistic. Of particular interest, here, are those psycholinguistic theories that have related syntactic predictions to syntactic complexity (see Levy, Fedorenko, \& Gibson, 2013 for a similar summary). These theories are known as memory-based theories and their main assumption is that each word input generates predictions regarding the constituents required to complete the current input string as a grammatical sentence. The longer these predictions have to be maintained, the greater is the processing difficulty due to memory limitations.

The Dependency Locality Theory (DLT, closely related to its predecessor, Syntactic Prediction Locality Theory: Gibson, 1998, 2000; Grodner \& Gibson, 2005) suggests that syntactic complexity and therefore sentence comprehension difficulties are associated with memory limitations: the processing resources involved in storage, retrieval and integration of syntactic representations are limited. To illustrate, consider the subject-extracted and object-extracted relative clauses (RC) in (1a) and (1b) respectively.

(1) a. The reporter who sent the photographer to the editor hoped for a story.

b. The reporter who the photographer sent to the editor hoped for a story.

(from Grodner \& Gibson, 2005)

Previous work has established that object-extracted relative clauses (ORC) are more difficult to process for normal adults than subject-extracted relative clauses (SRC) (see Ford, 1983; Gordon, Hendrick, \& Johnson, 2001; Grodner \& Gibson, 2005; King \& Just, 1991; Traxler, Morris, \& Seely, 2002 among may others; see also Friederici, Steinhauer, Mecklinger, \& Meyer, 1998 for German and Mak, Vonk, \& Schriefers, 2002, 2006 for Dutch). These studies have also demonstrated that the processing difficulty is located at the ORC verb (i.e. sent in ((1b)) (Grodner \& Gibson, 2005). According to the DLT, the key operation in syntactic comprehension is the storage and retrieval of predictions regarding the categories required to complete a given input string as a grammatical sentence and the integration of incoming input words with the partial structure built so far. Under this theory, the processing resources involved in storage, retrieval and integration of syntactic representations are limited. Therefore, dependency integrations are more difficult to process when more elements need to be integrated at the same time and/ or when there is long linear distance between the element that needs to be retrieved and the integration site. Thus, the DLT successfully predicts more processing difficulty associated with (1b) and more specifically the ORC verb sent, with which both the preceding subject and object NPs must be simultaneously integrated. 
A closely related memory-based theory is the Activation and Cue-Based Retrieval theory of Lewis and Vasishth (2005; see also Bartek, Lewis, Vasishth, \& Smith, 2011; Lewis, Vasishth, \& Van Dyke, 2006; Vasishth \& Lewis, 2006). According to this theory, sentence processing is incremental; linguistic input is fully integrated with previously processed material as soon as it is available. Similar to the DLT, establishing syntactic dependencies requires storage of preceding syntactic elements. Once an element is stored in memory, it goes out of the focus of attention and its activation level is subject to decay; increasing the distance between two dependent elements results in increasing processing difficulty because a distant element decays over time. A distinguishing feature of this theory is that under some circumstances, intervening materials, such as adverbs and prepositional phrases, can actually reactivate a distant element and facilitate its integration by counteracting decay (Vasishth \& Lewis, 2006). However, because retrieval is cue-based, it may be error-prone when the intervening material has features in common with the retrieval target. This phenomenon is known as similarity-based interference and it predicts the English SRC/ORC processing difficulty asymmetry in (1) because in the ORC case reporter and photographer need to be retrieved at the $\mathrm{RC}$ verb and since they share several features (i.e. both are animate, singular and definite), they interfere with one another.

To summarize, predictions about specific upcoming words and structures play a dominant role in sentence processing and can hinder sentence comprehension. The present study investigates whether PWA are able to build syntactic predictions and effectively use them in sentence processing. Specifically, we compare the processing of predictable syntactic dependencies with processing of unpredictable dependencies. With a predictable dependency, the syntactic parser detects the presence of a filler (usually, in the left periphery of the sentence) and prepares for the insertion of a gap (or trace) further downstream. This predictive process has been shown to be resource demanding and, according to the Complexity Threshold Hypothesis, might be associated with increased risk of processing breakdown. Unpredictable dependencies, by contrast, do not require syntactic prediction and should therefore be considerably less resource demanding. As a result, such dependencies should be less prone to give rise to parsing failure in PWA. Our hypothesis is schematically presented in Figure 1.

To our knowledge, there are only two previously published studies that explicitly investigate agrammatic patients' ability to predict upcoming events in sentence processing. Using a visual-world eye-tracking paradigm, Mack and colleagues (2013) explored whether verb meaning can be used by agrammatic patients to predict and facilitate the integration of the following noun. Several studies with young adult participants have shown that healthy listeners make anticipatory fixations to a target object, such as a cake within a scene containing other non-edible object, when processing a restrictive verb such as eat (i.e. a verb that has the potential to combine semantically with only one of the objects in the visual display), but not when processing an unrestrictive verb such as move (i.e. a verb that could combine with any of the objects in the display) (e.g. Altmann \& Kamide, 1999, 2007). Mack and colleagues tested healthy older adults and agrammatic patients in two experiments. In 


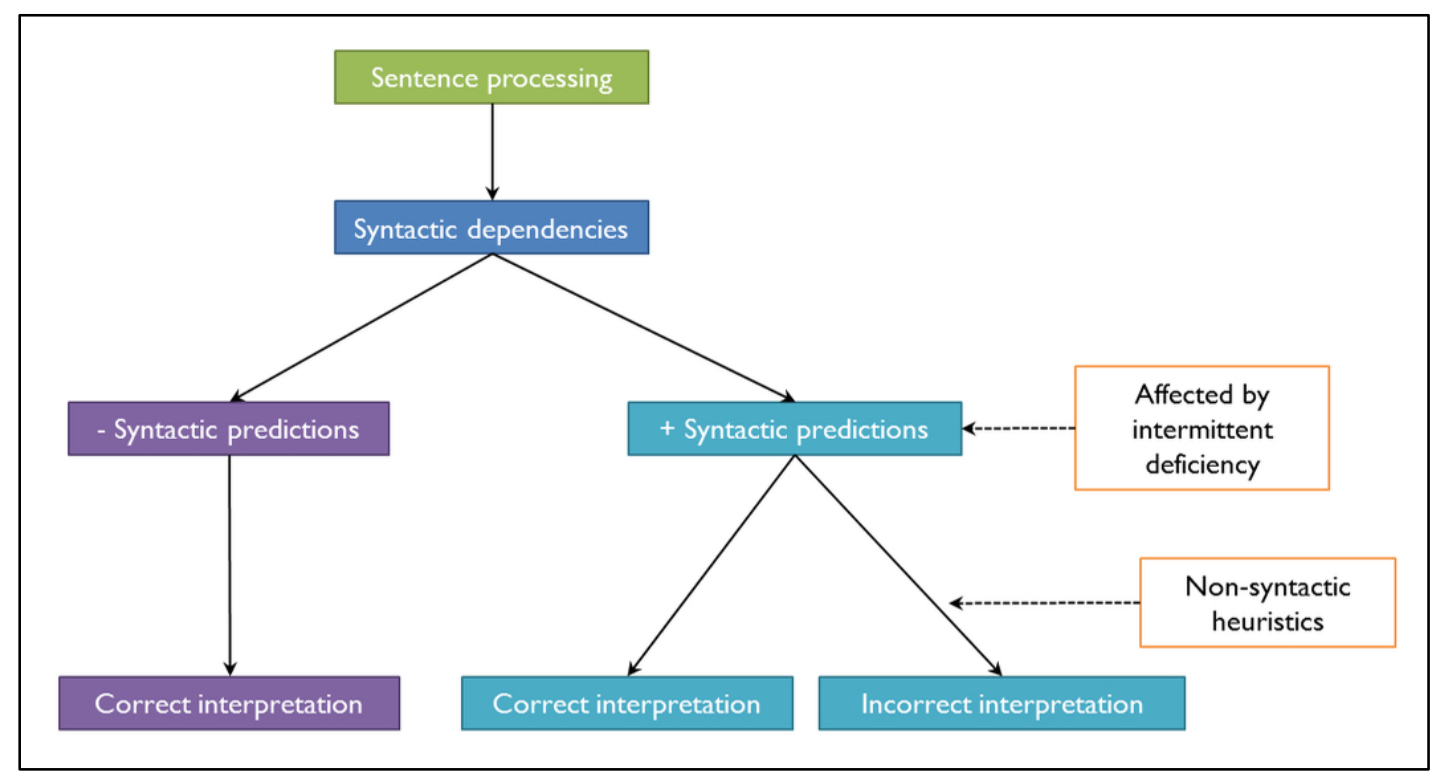

Figure 1 The Complexity Threshold Hypothesis: an account of sentence comprehension deficits in agrammatism.

their first experiment they explored eye movement patterns while processing full sentences with restrictive and unrestrictive verbs (e.g. Tomorrow Susan will open/break the jar), whereas in their second experiment the object noun was omitted to allow for a bigger time-window for predictions (e.g. Tomorrow Susan will open/break the...). In both experiments, participants were presented with visual displays containing four objects (e.g. jar, plate, stick, pencil) while listening to the sentences. The results of the first experiment showed that both healthy older adults and agrammatic patients failed to exhibit anticipatory fixations to the target object during presentation of the verb. In contrast with young adults in previous studies, participants in this study did not use the meaning of the verb to predict the upcoming argument prior to its onset. In the second experiment, healthy adults exhibited increased fixations to the target object $500 \mathrm{~ms}$ after the restrictive verb offset, whereas agrammatic patients exhibited a substantial delay (i.e. 1000-1500 ms after verb offset). Taken together, the results of Mack and colleagues' study suggest that lexicalsemantic prediction processes are slowed down in healthy aging and in agrammatism. In a recent study Hanne and colleagues (2015) investigated whether PWA are capable of using morphological cues to generate predictions during sentence processing using the visual-world paradigm. Specifically, they looked at the online processing of casemarking cues in case-unambiguous German SVO and OVS sentences as well as the online processing of a number-marking cue at the verb in case-ambiguous SVO and OVS sentences. PWA exhibited impairments in predictive processes based on morpho-syntactic cues, which, according to the authors, suggests that PWA's sentence processing relies on a wait-and-see strategy and initiate predictions at a later stage compared to controls. 


\subsection{Aim of the study and predictions}

The aim of the present study is to combine aphasia-specific accounts and psycholinguistic accounts in explaining sentence comprehension deficits in aphasia. Specifically, we use syntactic predictions, known to be resource demanding, as a proxy to investigate the effect of resource limitations on sentence processing in PWA. Our hypothesis is that PWA's syntactic parser occasionally fails due to patients' resource limitations, and that these failures are most likely to occur when syntactic complexity exceeds a certain threshold (Complexity Threshold Hypothesis). To explore the Complexity Threshold Hypothesis we devised two experiments that investigate two types of syntactic dependencies.

Experiment 1 investigates processing of unpredictable syntactic dependencies, that is, dependencies whose establishment does not require comprehenders to generate and use syntactic predictions based on syntactic cues such as active fillers. To illustrate, Experiment 1 explores processing of ambiguous doubly quantified sentences, such as $A$ boy is photographing every girl. The first interpretation of a sentence like this is that there is a single boy who photographs all the girls in the relevant domain. In this interpretation, the two quantified phrases (i.e. $A$ boy and every girl) keep their surface order and the interpretation itself is called the surface scope interpretation. The second interpretation is that for each girl in the relevant domain there is some different boy that is photographing that girl. This interpretation is called the inverse scope interpretation and requires comprehenders to invert the order of the two quantified phrases; the lower quantified phrase (i.e. every girl) must take scope over the higher quantified phrase (i.e. A boy). This phenomenon is known as scope inversion and happens through a covert movement operation that takes place in the Logical Form. Similar to overt movement, covert movement results in a dependency. However, this dependency is unpredictable, since there is no syntactic cue that could signal the dependency and generate a syntactic prediction. We predict that PWA will be able to assign both scope interpretations to ambiguous doubly quantified sentences. Although the inverse scope interpretation requires the establishment of a dependency, this dependency is unpredictable and therefore less resource demanding compared to predictable dependencies. Previous psycholinguistic studies that have explored processing of scope ambiguity in neurologically intact subjects have shown that those subjects often exhibit biases of quantifier scope interpretation, that is, they arrive at specific scope interpretations more easily compared to other interpretations (e.g., Anderson, 2004; Kurtzman \& MacDonald, 1993; Tunstall, 1998). Nevertheless, there are studies suggesting that there are no scope biases (Dwivedi, Phillips, Einagel, \& Baum, 2010). We therefore have no specific predictions regarding scope biases. Our speculation is that scope preferences might result from interference effects that are stronger in participants with intact memory, and that therefore they will be more evident in the controls group. The way we interpret scope preferences is that, although the context might prime a specific interpretation, comprehenders are still able to build in parallel the alternative interpretation and make their choice (in a truth-value judgment task, for instance) 
based on which interpretation is their preferred one. We revisit this idea in section 2.3 where we discuss the findings of Experiment 1.

Experiment 2 explores the possible dissociation between predictable and unpredictable dependencies using the minimal pair of sentences with moved contrastive focus and sentences with in situ contrastive focus. To illustrate, sentences with moved contrastive focus like (2a) contain a predictable scope-marking filler-gap dependency, namely the dependency between the contrastively focused constituent THE MAN that has been moved to the left periphery, and its base-generated position (i.e. the post-verbal, object position marked with $\square$ ). This dependency is predictable; the contrastively focused constituent acts as a syntactic cue generating a syntactic prediction.

(2) a. THE MAN the woman is washing $\square$, not the boy.

b. The woman is washing THE MAN, not the boy.

In sentences with in situ contrastive focus like (2b), covert movement of the contrastively focused constituent THE MAN to the left periphery is required, in order to assign it its scope. This operation is similar to the one taking place when assigning inverse scope interpretation to ambiguous doubly quantified sentences. It therefore results in a dependency, which is unpredictable, since there is no syntactic cue generating a syntactic prediction. Based on the psycholinguistic evidence presented in the previous section, we hypothesize that sentences with moved contrastive focus are more resource demanding compared to sentences with in situ contrastive focus, because of the presence of predictable dependencies in the former. We therefore predict that sentences with moved contrastive focus will elicit more errors compared to sentences with in situ contrastive focus. We further predict that this dissociation will be more prominent in the group of agrammatic patients. Processing of sentences with contrastively focused constituents (either moved or in situ) requires the integration of syntactic and prosodic knowledge (alongside contextual knowledge), and therefore might be resource demanding. We thus predict that PWA will not perform at ceiling when processing sentences with in situ contrastive focus, despite the absence of syntactic predictions, due to the presence of contrastive intonation.

\section{Experiment 1}

The aim of Experiment 1 is to explore the hypothesis that PWA have no difficulties in processing sentences with unpredictable intra-sentential dependencies. This will be achieved by exploring processing of ambiguous doubly quantified sentences as in (3).

(3) A boy is photographing every girl.

a. $\exists x[\operatorname{boy}(\mathrm{x}) \& \forall \mathrm{y}[\operatorname{girl}(\mathrm{y})->$ photograph $(\mathrm{x}, \mathrm{y})]]$ [read as: "There is a boy $\mathrm{x}$ and for all $\mathrm{y}$, if $\mathrm{y}$ is a girl, $\mathrm{x}$ is photographing $\mathrm{y}$ ]

b. $\forall \mathrm{x}[\operatorname{girl}(\mathrm{x})->$ ヨy $[\mathrm{boy}(\mathrm{y}) \&$ photograph $(\mathrm{y}, \mathrm{x})]]$ [read as: "For every $\mathrm{x}$, if $\mathrm{x}$ is a girl then there exists a boy $\mathrm{y}$, such that $\mathrm{y}$ is 


\section{photographing $\mathrm{x}]$}

Most speakers of English agree that (3) has two interpretations. The first interpretation is that there exists a single boy who photographs all the girls in the relevant domain. This interpretation is called the surface scope interpretation. The second possible interpretation is that some different boy is photographing each girl. This interpretation is called the inverse scope interpretation. For a representation of the two interpretations using logical notation see (3a) and (3b) respectively.

Structures like (3) are of particular interest, because the inverse scope interpretation is the result of a covert operation similar to movement. Specifically, it has been argued that in order to arrive at the inverse scope interpretation the lower quantified phrase (i.e. every girl) must take scope over the higher quantified phrase (i.e. A boy) by covertly moving to the left periphery of the sentence (see May, 1977 and subsequent work). This operation is known as Quantifier Raising (QR) and results in an unpredictable dependency, since there are no syntactic cues (e.g. active filler) as in overt movement. Exploring processing of ambiguous doubly quantified sentences therefore allows us to investigate PWA's performance when the establishment of intra-sentential dependencies does not require syntactic predictions.

\subsection{Methods}

\subsubsection{Participants}

Seven Greek-speaking PWA and 18 healthy young and age-matched adults participated in the experiment. All participants were (premorbidly) right-handed, with normal or corrected-to-normal vision and gave informed consent. PWA had an average age of 53.47 years ( $\mathrm{SD}=7.87$; range $42-62$ years) and an average of 12.29 years of education ( $\mathrm{SD}=3.30$; range: $6-16$ years). Healthy adults had an average age of 36.94 years $(\mathrm{SD}=15.55$; range: $21-63$ years) and an average of 16.17 years of education ( $\mathrm{SD}=4.09$; range: 6 - 20 years). Error! Reference source not found. presents demographic data for all participants. Detailed lesion information was available for five participants (all but P5 and P6) (see Table 1 in the APPENDIX). PWA were diagnosed as having aphasia by the second author on the basis of their performance on the Greek version of the Boston Diagnostic Aphasia ExaminationShort Form (BDAE-SF; Goodglass \& Kaplan, 1972) adapted in Greek (Tsapkini, Vlahou, \& Potagas, 2009).

Table 1 Participants' demographic details.

\begin{tabular}{cccccccc} 
ID & Sex & Age & Education & TPO$^{1}$ & Diagnosis & Hemiplegia & Profile \\
\hline P1 & M & 42 & $14 y r s$ & 4.1 yrs & Stroke & Right & Non-fluent, agrammatic \\
P2 & M & 48 & $15 y r s$ & $6.8 y r s$ & Stroke & Right & Non-fluent, agrammatic \\
P3 & M & 58 & 12 yrs & $5.5 y r s$ & Stroke & No & Non-fluent, agrammatic \\
P4 & M & 46 & $6 y r s$ & $3.2 y r s$ & Stroke & Right & Non-fluent, agrammatic \\
P5 & M & 62 & $16 y r s$ & $0.6 y r s$ & Stroke & No & Non-fluent, non-agrammatic \\
P6 & M & 59 & 11 yrs & $3.4 y r s$ & Stroke & No & Non-fluent, non-agrammatic \\
P7 & M & 59 & $12 y r s$ & $4.8 y r s$ & Stroke & No & Non-fluent, non-agrammatic \\
\hline C1 & M & 21 & $15 y r s$ & & & & \\
C2 & F & 22 & $16 y r s$ & & & &
\end{tabular}




\begin{tabular}{lllll} 
C3 & F & 22 & $16 \mathrm{yrs}$ & \\
$\mathrm{C} 4$ & $\mathrm{~F}$ & 23 & $16 \mathrm{yrs}$ \\
$\mathrm{C} 5$ & $\mathrm{~F}$ & 23 & $16 \mathrm{yrs}$ \\
$\mathrm{C} 6$ & $\mathrm{~F}$ & 25 & $18 \mathrm{yrs}$ & \\
$\mathrm{C} 7$ & $\mathrm{~F}$ & 25 & $16 \mathrm{yrs}$ & \\
$\mathrm{C} 8$ & $\mathrm{~F}$ & 30 & $19 \mathrm{yrs}$ & $\mathrm{N} / \mathrm{A}$ \\
$\mathrm{C} 9$ & $\mathrm{~F}$ & 32 & $18 \mathrm{yrs}$ & \\
$\mathrm{C} 10$ & $\mathrm{~F}$ & 34 & $17 \mathrm{yrs}$ & \\
$\mathrm{C} 11$ & $\mathrm{~F}$ & 36 & $16 \mathrm{yrs}$ & \\
$\mathrm{C} 12$ & $\mathrm{~F}$ & 36 & $22 \mathrm{yrs}$ & \\
$\mathrm{C} 13$ & $\mathrm{~F}$ & 37 & $16 \mathrm{yrs}$ & \\
$\mathrm{C} 14$ & $\mathrm{~F}$ & 55 & $23 \mathrm{yrs}$ \\
$\mathrm{C} 15$ & $\mathrm{M}$ & 59 & $9 \mathrm{yrs}$ & \\
$\mathrm{C} 16$ & $\mathrm{~F}$ & 60 & $6 \mathrm{yrs}$ & \\
$\mathrm{C} 17$ & $\mathrm{~F}$ & 62 & $12 \mathrm{yrs}$ \\
$\mathrm{C} 18$ & M & 63 & $20 \mathrm{yrs}$ & \\
\hline
\end{tabular}

Language testing included a narrative speech sample and comprehension of syntactically complex sentences. The aim of the narrative speech analyses was to assess the quality of speech produced by PWA in terms of fluency, grammaticality, complexity of syntactic structure, and use of grammatical morphemes. Narrative speech samples were obtained from conversation about the stroke incident and description of the Cookie Theft picture from the BDAE (Goodglass \& Kaplan, 1972) and the Picnic scene from the Western Aphasia Battery (WAB, Kertesz, 1982). Speech samples were analyzed with the Quantitative Production Analysis protocol (QPA; Saffran, Berndt, \& Schwartz, 1989; Varkanitsa, 2012). All PWA had nonfluent speech production (mean words per minute: 17.17, range: $12-25.15$ words per minute). Four patients (P1, P2, P3 and P4) also presented agrammatic speech, characterized by selective omission of closed class words and overall reduction of grammatical morphemes, both free (mainly, of determiners and prepositions) and bound (i.e. verb inflections). From now on, these PWA will be referred to as agrammatic patients. Apart from P3, the agrammatic patients produced predominantly nouns and had difficulties in generating sentences. When they managed to do so, their sentences were either ill-formed (i.e. contained morpho-syntactic errors) or were structurally simple (i.e. contained only the minimal constituents, a verb and an objectDP). In contrast, P5, P6 and P7 showed only occasional omission of grammatical morphemes and had less difficulty in generating simple grammatical sentences. These PWA will be referred to as non-agrammatic patients.

PWA's syntactic comprehension abilities were assessed with a picture-pointing task testing comprehension of $w h$-questions and a sentence-picture matching task testing comprehension of passives and relative clauses. The procedure in the picturepointing task was similar to the task of Thompson and colleagues (1999). Each picture depicted a reversible action performed by three animates (persons or animals). Each trial consisted of one picture and one question read aloud by the experimenter, and PWA were asked to point to the person or the animal representing the answer to the question. Questions were repeated upon request. There were four conditions: whosubject questions, who-object questions, which-NP-subject questions and which-NP- 
object questions. The sentence-picture matching task consisted of three conditions: passive sentences, subject-relative clauses and object-relative clauses. Each trial included one sentence paired with two pictures. PWA were instructed to choose the picture that correctly described the sentence heard. The agrammatic patients presented impaired comprehension of syntactically complex sentences (passives, relative clauses and wh-questions), whereas the non-agrammatic patients performed close to normal. For results of individual PWA see Table 2 in the APPENDIX.

\subsubsection{Stimuli}

A truth-value judgment task was designed to investigate processing of scope ambiguities. The materials consisted of single sentences presented simultaneously with pictures. Stimulus sentences were spoken by a female native speaker of Greek at a natural rate and with the type of intonation that is typical for Greek "all new" affirmative declarative sentences, where no word carries narrow focus. Stimuli were recorded in a soundproof recording booth at UCL's Chandler House research laboratory. Any noise before the onset and after the offset of the sentences was removed using Audacity (http://audacity.sourceforge.net/). A set of 133 pictures was designed by modifying images from royalty free clip art available on the Internet. The size of the pictures was $900 \times 612$ pixels.

The experimental items consisted of 20 ambiguous, doubly quantified sentences, such as (4), used in four experimental conditions.

$$
\begin{aligned}
& \text { Mia gineka fotografizi kathe pedi } \\
& \text { A } \text { woman }_{\mathrm{NOM}} \text { photograph }_{3 \mathrm{rd} \text { SING }} \text { every } \text { child }_{\mathrm{ACC}} \\
& \text { 'A woman is photographing every child' }
\end{aligned}
$$

All sentences contained the quantifiers "a" and "every" in the subject-DP and the object-DP, respectively. In total, there were five words in a simple SVO word order. Each sentence was paired with two pictures, one that required a surface scope interpretation of the sentence and another that required an inverse scope interpretation, resulting in 40 target sentence-picture pairs. The same 20 sentences were also paired with pictures deviating from the truth conditions of the sentence, resulting in 40 negative items. The reason for including these items was to force participants to look carefully at each picture, with particular attention to the truth conditions imposed by the quantifiers, and most importantly, to counterbalance the number of YES and NO responses.

Overall, we had a crossed two-factor two level design. The first factor is the interpretation. The two levels of interpretation are surface scope and inverse scope. The second factor is matching. The two levels of matching are match (i.e. picture does not deviate from the truth conditions of the sentence) and mismatch (i.e. picture deviates from the truth conditions of the sentence). This design resulted in 80 experimental stimuli divided between the four different combinations; 20 surface scope - match stimuli, 20 inverse scope - match stimuli, 20 surface scope - mismatch stimuli and 20 inverse scope - mismatch stimuli. Apart from the experimental stimuli, 
we also had 60 fillers, including relative clauses and passive sentences, and 13 practice items, resulting in an overall of 153 trials.

Stimuli were divided between two parts of 70 trials (i.e. 40 experimental and 30 filler), which we call "subtests", so that the same item (i.e. sentence-picture pair) only appeared once in a single session and there was no repetition. There were an equal number of left-to-right and right-to-left action depictions. Each participant received a different order of the items in a "random with a rule" manner (i.e. no more than three items of the same condition appeared together). Figure 2 provides a pictorial exemplification of the design.

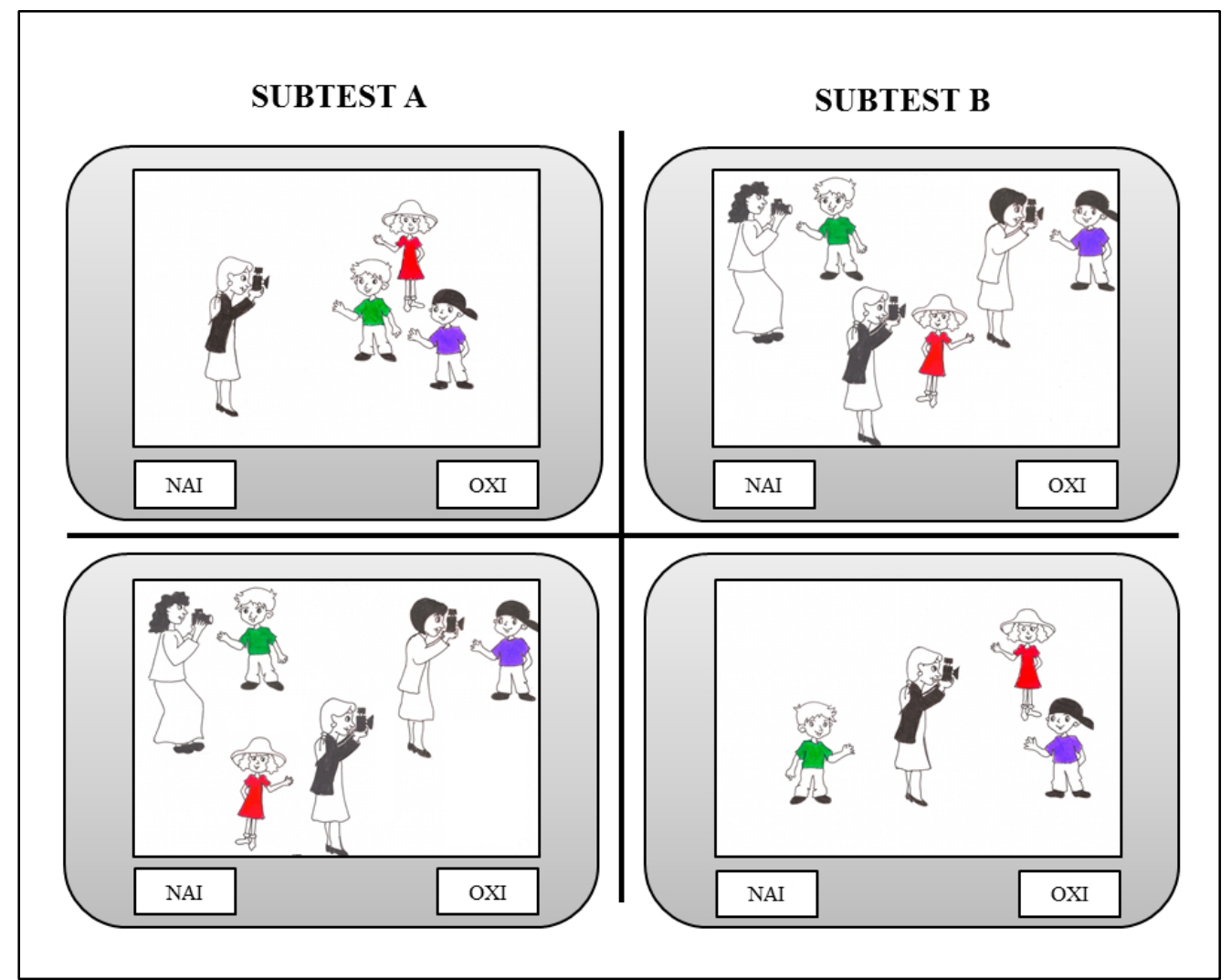

Figure 2 Sample stimulus images for the experimental sentence $A$ woman is photographing every child. The top left picture illustrates the surface scope - match condition, the top right picture illustrates the inverse scope match condition, the bottom left picture illustrates the inverse scope - mismatch condition and the bottom right picture illustrates the surface scope - mismatch condition.

\subsubsection{Procedure}

After providing informed consent, participants were seated in a quiet testing room, in front of a computer. Stimuli were presented on a Dell laptop computer with a 15" screen using the SyntaxLab program developed for this study. Prior to the start of the experiment, the template for the first part of the trials was presented and the instructions were given to participants. When ready, participants pressed the spacebar and the first trial began. The picture appeared at the center of the screen and the spoken sentence was simultaneously heard via two speakers. Below the picture there were two labels: NAI (YES) and OXI (NO). At the end of the spoken sentence a yellow square appeared on the screen, at which point participants responded as 
quickly as they could by pressing one of the two response keys on the keyboard. The picture remained visible until participants responded. RTs and accuracy data were obtained from the offset of the spoken sentence to the key press. Responses made too early were ignored. For patients with aphasia, a new trial began when the experimenter clicked on the next button in the right corner of the screen. For the control group, a new trial began immediately after their response to the previous trial. Participants undertook a thirteen-item practice test prior to beginning the experiment. Stimuli were divided into two subtests. Every participant performed both subtests. For the patients group there was a break of 30 minutes between the two subtests, during which the patients performed other tasks. For the group of controls the break was shorter. The instructions were repeated before the second subtest for each participant.

\subsubsection{Data analysis}

We analyzed response accuracy using generalized linear mixed models (GLMMs), with the binomial distribution and logit link function. All GLMM analyses were run using the lme4 package (Bates, Maechler, Bolker, \& Walker, 2013) in R (R Core Team, 2013). Initially a model was fitted with a random intercept for participants $(\mathrm{N}$ $=25$ ) and for items, that is the different scenarios described in sentences (e.g. kissing, kicking, shooting etc.) $(\mathrm{N}=20)$, and fixed effects for group (i.e. agrammatic patients, non-agrammatic patients and controls), interpretation (i.e. surface scope and inverse scope) and matching (i.e. match and mismatch). A three-way interaction between the fixed factors was initially added. However, this complicated model failed to converge and we therefore run a model with three two-way interactions; an interaction between group and interpretation, an interaction between group and matching and an interaction between interpretation and matching. Age and Educational Level were also included in the models as covariates. Since these variables lack natural zeroes and in order to interpret them more easily, we centered them to their means (Cohen \& Cohen, 1983). The statistical analyses on RTs were carried out on log-transformed values in order to satisfy the assumptions of additivity and linearity (see Gelman $\&$ Hill, 2007). We report coefficient estimates, their standard errors, $z$-scores and pvalues.

\subsection{Results}

\subsubsection{Accuracy}

Figure 3 presents the mean values for correct responses per group along with 95\% confidence intervals. The statistical analysis revealed a main effect of group (loglikelihood ratio $\left.[\mathrm{LLR}] \chi^{2}(2)=10.36, p<0.01\right)$. Agrammatic patients were more likely to respond correctly compared to controls (coefficient $=0.92, \mathrm{SE}=0.44, z=2.07, p$ $<0.05$ ). The non-agrammatic patients also performed significantly better than controls (coefficient $=1.88, \mathrm{SE}=0.56, z=3.36, p<0.001$ ). The difference in performance between the agrammatic and the non-agrammatic patients was not significant (coefficient $=-0.68, \mathrm{SE}=0.75, z=2.11, p=0.37$ ).

There was also a main effect of interpretation $\left(\operatorname{LLR} \chi^{2}(1)=13.39, p<0.001\right)$. Overall, participants were more likely to respond correctly to trials requiring the 
inverse scope interpretation than trials requiring the surface scope interpretation (coefficient $=0.46, \mathrm{SE}=0.13, z=3.66, p<0.001$ ). Finally, the main effect of education level was significant $\left(\operatorname{LLR} \chi^{2}(1)=4.88, p<0.05\right)$; that is, participants with higher educational level performed better. The main effect of matching was not significant $\left(\operatorname{LLR} \chi^{2}(1)=0.25, p=0.61\right)$ nor was the main effect of age (LLR $\chi^{2}(1)=$ $0.96, p=0.33)$.

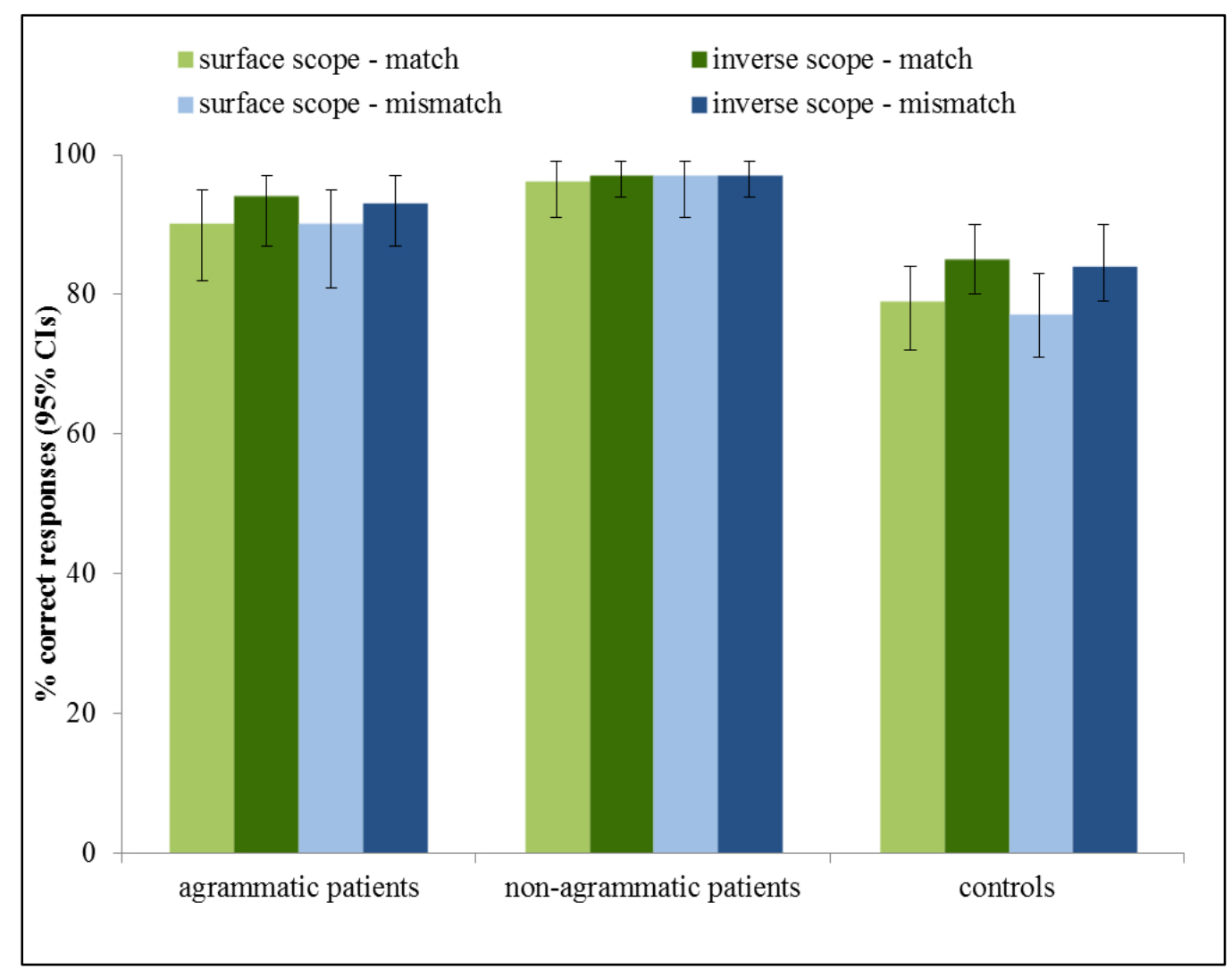

Figure 3 Comprehension accuracy as a function of condition and participants group, means (\%) and 95\% confidence intervals.

Considering the three two-way interactions, the interaction between group and interpretation was statistically significant $\left(\operatorname{LLR} \chi^{2}(2)=18.36, p<0.001\right)$. Trials associated with inverse scope interpretations elicited significantly more accurate responses compared to trials associated with surface scope interpretations. This performance pattern was more likely to occur in the group of controls compared to both agrammatic (coefficient $=1.55, \mathrm{SE}=0.44, z=3.52, p<0.001$ ) and nonagrammatic $($ coefficient $=1.59, \mathrm{SE}=0.78, z=2.03, p<0.05)$ patients. The interaction between group and matching was not significant $\left(\operatorname{LLR} \chi^{2}(2)=5.13, p=0.08\right)$ nor was the interaction between interpretation and matching $\left(\operatorname{LLR} \chi^{2}(1)=0.04, p=0.85\right)$.

Individual data supports the results of group analysis (see Figure 4). There was no great variability within the patient group for most conditions. However, it is notable that P1, from the group of agrammatic patients, exhibited a strong scope preference in the match conditions. Though he was able to assign inverse scope interpretations to matching pictures, his performance on the surface scope - match condition was very low. Finally, P4, also from the agrammatic group, and P6, from 
the non-agrammatic group, performed around $50 \%$ in the surface scope - mismatch condition.

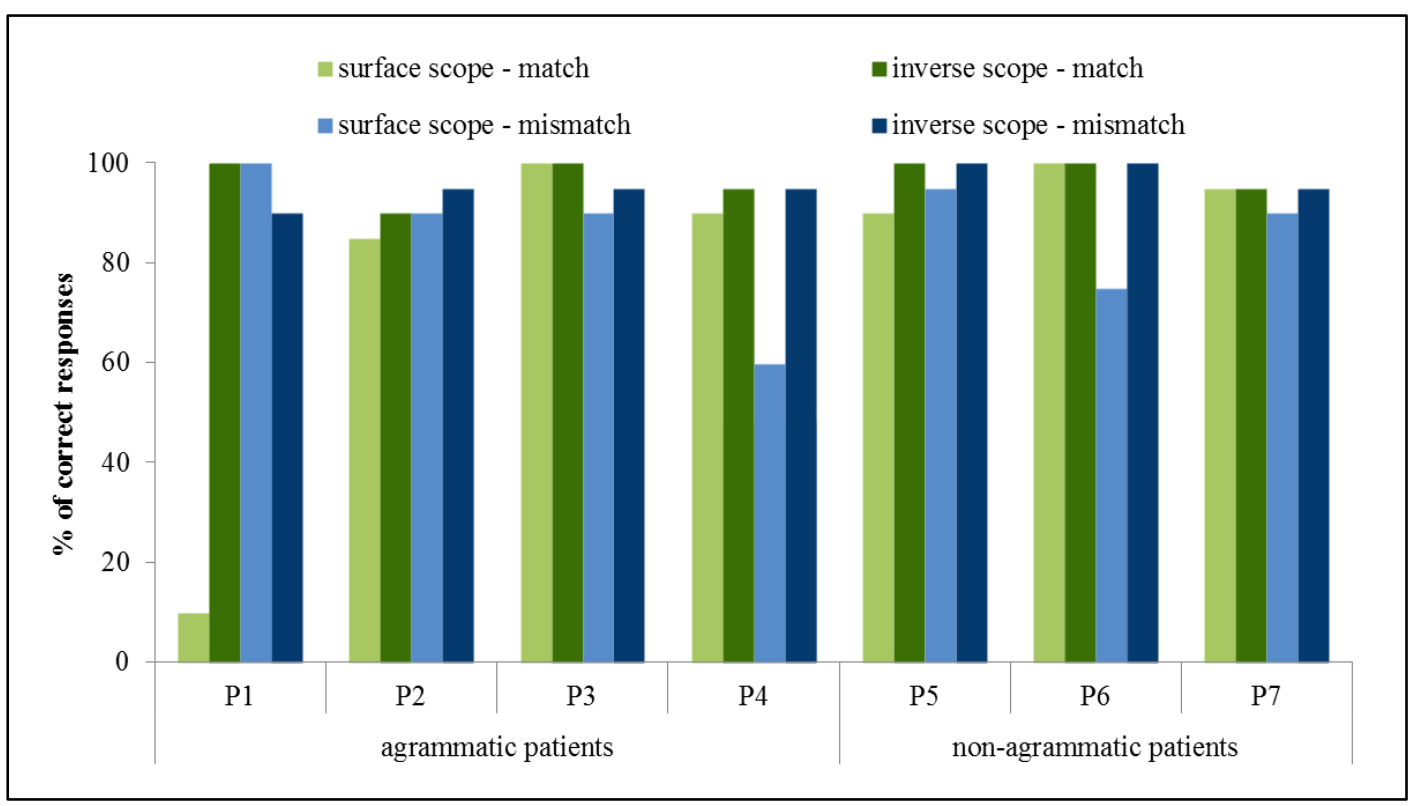

Figure 4 Individual comprehension accuracy, means (\%).

\subsubsection{Reaction times}

Log-transformed values of RT data for accurate responses were entered as the dependent variable into a generalized linear mixed model. Overall, there was a main effect of group (LLR $\left.\chi^{2}(2)=18.22, p<0.001\right)$; both the agrammatic and the nonagrammatic patients exhibited slower RTs compared to controls (see Figure 5). There was also a main effect of interpretation $\left(\operatorname{LLR} \chi^{2}(1)=4.34, \mathrm{p}<0.05\right)$. Overall, participants were faster in trials associated with surface scope interpretations compared to trials associated with inverse scope interpretations. The main effect of matching was also statistically significant $\left(\operatorname{LLR} \chi^{2}(1)=5.10, \mathrm{p}<0.05\right)$; Participants were slower in trials where sentences were associated with mismatching pictures compared to trials where sentences were associated with matching pictures. Individual comparisons for these main effects did not reach significance.

Considering the three two-way interactions, only the interaction between group and matching was statistically significant $\left(\operatorname{LLR} \chi^{2}(2)=9.54, p<0.01\right)$. Both the agrammatic and the non-agrammatic patients were significantly slower in the mismatching conditions compared to matching conditions, whereas the group of controls performed similarly across conditions. Again, individual comparisons did not reach significance. Finally, the interaction between group and interpretation was not significant $\left(\operatorname{LLR} \chi^{2}(2)=0.22, \mathrm{p}=0.89\right)$ nor was the interaction between interpretation and matching $\left(\operatorname{LLR} \chi^{2}(1)=1.96, \mathrm{p}=0.16\right)$.

Individual data inspection revealed great variability within the groups of patients with aphasia (see Figure 6). Considering the agrammatic patients, P3 and P4 exhibited longer RTs in the mismatch conditions compared to match conditions. However, P3 exhibited longer RTs when assigning inverse scope interpretation to matching pictures than assigning inverse scope interpretations to the same pictures 
(see also P1), whereas P4 exhibited longer RTs when assigning inverse scope interpretations to matching pictures than assigning surface scope interpretations to the same sentence (see also P2). As for the non-agrammatic patients, P6 exhibited longer RTs compared to the other two patients for all conditions. P5 exhibited slightly longer RTs for the negative items compared to positive items, whereas P7 performed roughly the same across all conditions.

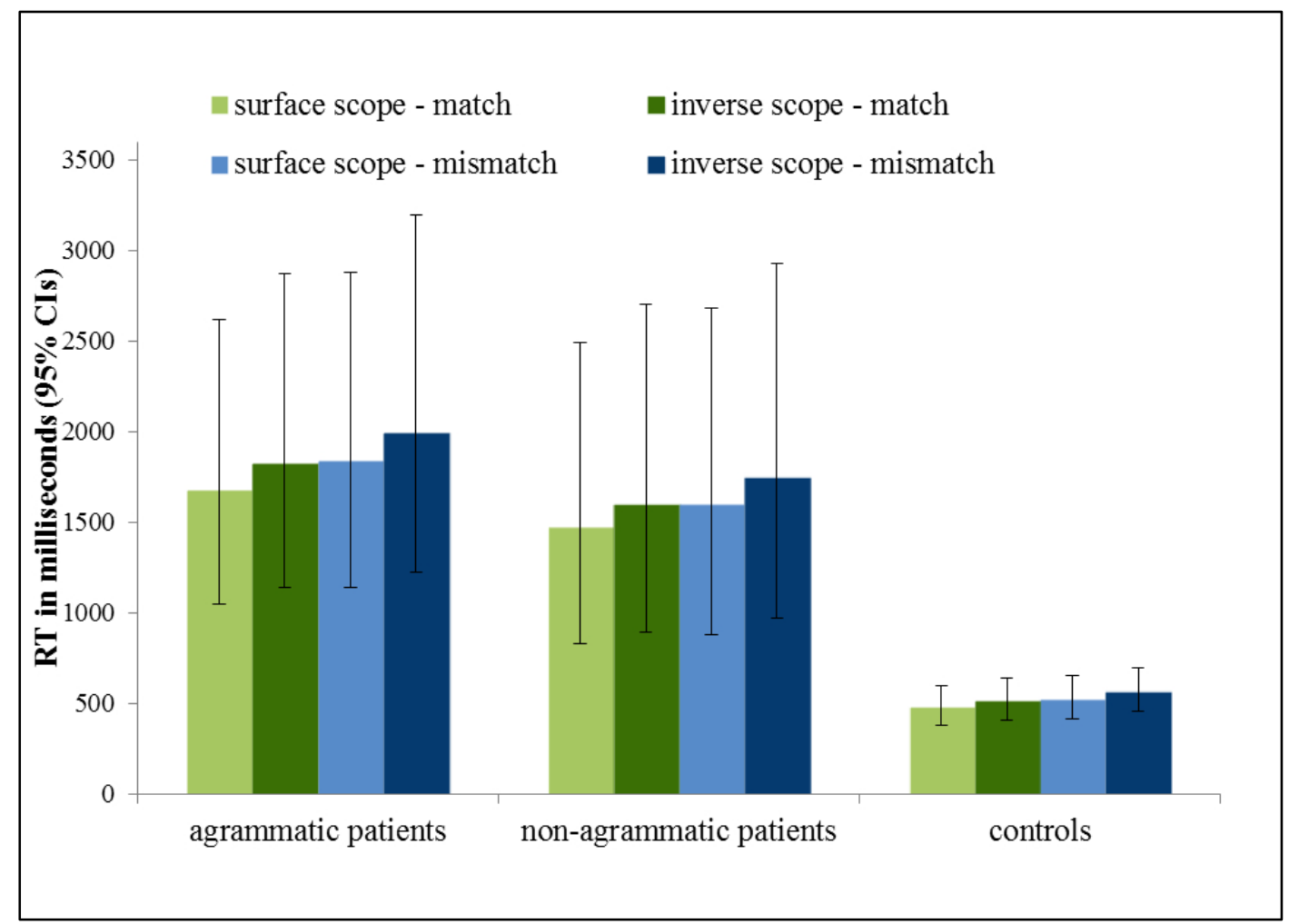

Figure 5 RTs in correct trials as a function of condition and participants group, milliseconds.

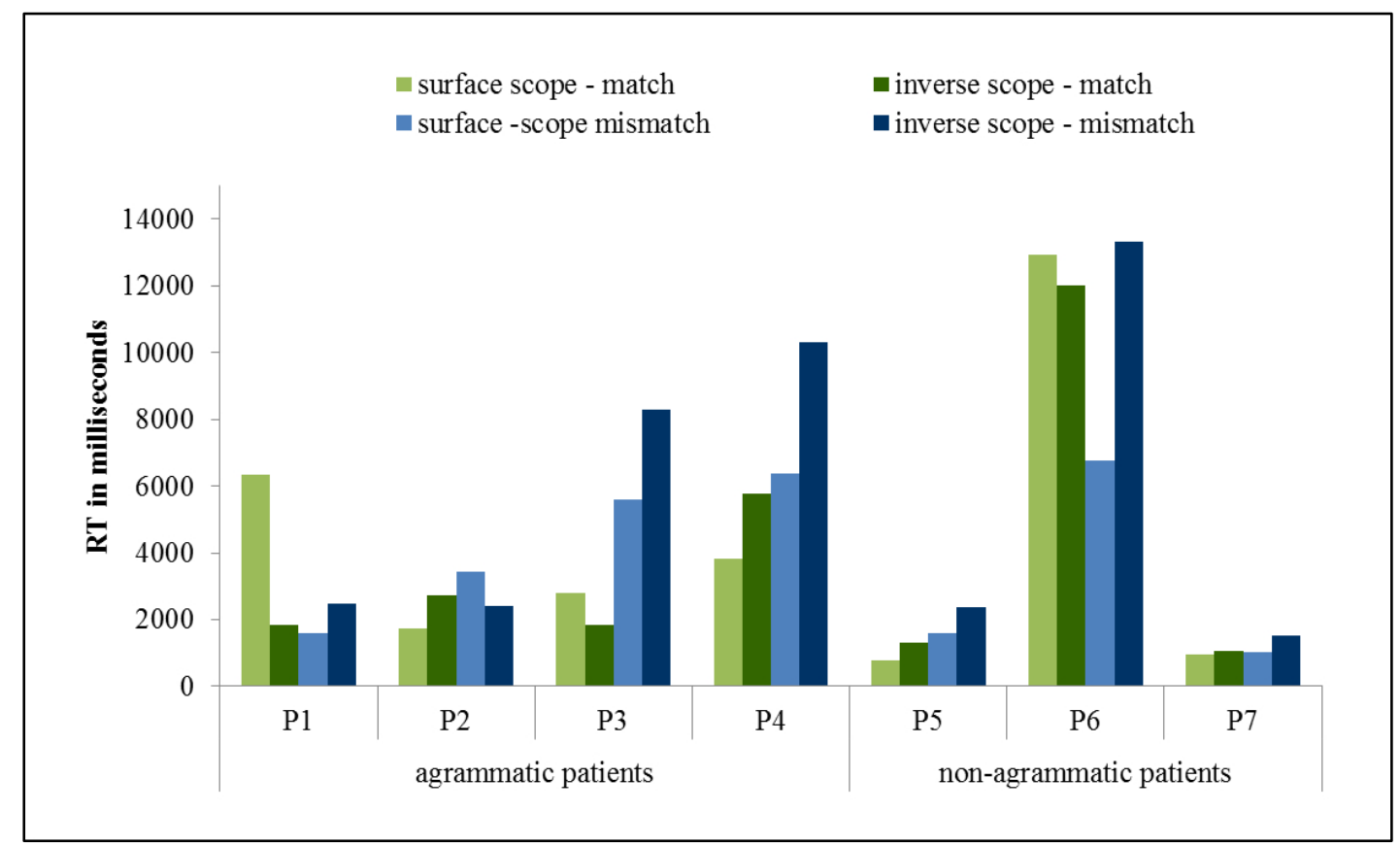

Figure 6 Individual RTs in correct trials, milliseconds. 


\subsection{Discussion}

Experiment 1 investigated processing of ambiguous doubly quantified sentences by Greek-speaking PWA. Our aim was to show that PWA have intact knowledge of QR. The background sentence comprehension assessment revealed that four patients were agrammatic, that is, their comprehension of movement-derived sentences (and therefore sentences with syntactic predictions) was impaired. Accurate response to trials associated with inverse scope interpretations would therefore provide evidence that when syntactic predictions are kept to a minimum PWA's sentence comprehension is intact, even in cases of patients suffering from agrammatism. Before discussing the findings for PWA, we will first review the results for the group of controls.

The most surprising finding regarding controls' performance was that overall they performed poorly compared to PWA. This could be attributed to the fact that processing of ambiguous sentences in general is associated with increased processing cost, which may have a negative impact on performance. In fact, a recent ERP study reports a slow negative shift elicited in continuation sentences following ambiguous contexts, which is very similar to the Late Slow Wave elicited when performing a conceptually difficult task (Dwivedi et al., 2010). Such an interpretation would, however, predict similar or even greater difficulties for PWA, which is not the case. Another plausible explanation for controls' performance pattern could be related to scope preferences. We suggest that controls exhibited biases towards specific interpretations when processing ambiguous doubly quantified sentences. As a group, they were more likely to endorse an inverse scope interpretation than a surface scope one. Having said that, individual data inspection revealed great within group variability. Specifically, two participants exhibited very strong preferences but towards opposite directions; one of them was unable to accept as correct pictures depicting surface scope interpretations, whereas the other one was unable to accept as correct pictures depicting inverse scope interpretations. Another four participants performed low in trials associated with surface scope interpretations (i.e., they exhibited an inverse scope preference) and another three performed low in trials associated with inverse scope interpretations (i.e., they exhibited a surface scope preference). The rest of the controls did not exhibit any scope preferences. We therefore argue that what looks like poor performance at least for the control group could be considered as the result of individual variability due to scope biases.

The idea that speakers exhibit scope biases when processing ambiguous sentences is not new. As early as in the 70 s and 80 s, researchers suggested that, depending on the type and/ or the linear order of the quantified phrases, speakers may present scope biases, that is, they may prefer surface scope interpretations over inverse scope interpretations and vice versa. To illustrate, Johnson-Laird (1969) proposed the linear order principle according to which scope preferences are determined through word order and surface scope interpretations should therefore be the preferred ones. A few years later, Ioup (1975) suggested that the picture is more complicated; quantifiers' lexical characteristics and quantified phrases' grammatical function interact to determine scope preferences (see quantifier hierachy principle 
and grammatical hierarchy principle in Ioup, 1975). In a similar vein, Fodor (1982) proposed that scope preferences depend on both linear order and quantifiers' characteristics. Specifically, when every precedes $a$, both linear order and quantifiers' characteristics favour a surface scope interpretation. However, when a precedes every, linear order favours a surface scope interpretation but the characteristics of every favour an inverse scope interpretation. In the latter case, Fodor suggests that speakers initially build a representation in which $a$ refers to one entity and, when encountering every, this interpretation is revised, incurring a processing cost. Kurtzman and MacDonald (1993) also proposed that scope resolution depends on the interaction of multiple principles. They argued that when these principles favour one representation, that representation is built. However, when principles are in conflict, the two representations are evaluated in parallel and compete for adoption, incurring a processing cost. The idea that assigning specific scope interpretations to ambiguous sentences is associated with a processing cost has also been proposed in more recent psycholinguistic studies. In a series of offline and real time experiments testing structures very similar to those examined in this experiment, Anderson (2004) showed that speakers arrive more easily at surface scope interpretations. This was reflected in both accuracy and reading time data. Based on these findings, Anderson suggested that speakers have a strong preference towards surface scope interpretations, which can be overridden when context supports inverse scope interpretations. This, however, incurs a processing cost (see Processing Scope Economy Principle in Anderson, 2004; see also Tunstall, 1998 for a similar approach). Apart from studies showing that speakers have scope biases when processing ambiguous doubly quantified sentences there are also studies suggesting that there are no scope preferences at all; at very early stages of processing doubly quantified sentences are underspecified regarding quantifier scope. In an event-related brain potentials study Dwivedi and colleagues (2010) showed that the parser does not immediately assign a logical meaning to ambiguous, doubly quantified sentences. Rather, scopally ambiguous sentences are left underspecified until further disambiguating information arrives (see also Raffray \& Pickering, 2010).

From the previous psycholinguistic evidence briefly presented above, we can conclude that there is no consensus in the literature as to the direction of scope biases, if any. In fact, thinking of the sentences tested in this experiment (i.e. a...every structures), the various scope-processing principles are in conflict. Johnson-Laird's and Anderson's principles would predict a preference towards surface scope interpretations, a performance pattern that was observed in four out of 18 control participants. Ioup's and Fodor's principles, on the other hand, would predict a preference towards inverse scope interpretations, a performance pattern that was observed in five out of 18 control participants. Finally, Dwivedi and colleagues' hypothesis that there are no scope preferences at all would predict speakers to assign both scope interpretations with equal facility, a performance pattern that was observed in the rest of the controls group. All in all, the surprising finding that controls performed poorly compared to PWA may be attributed to the individual variability observed in the controls group, which is, however, consistent with the varying set of 
scope-processing principles reported in this section. Before moving into the discussion of PWA's performance pattern, it is worth noting that reaction time data do not reflect any scope biases. This is probably due to the fact that we measured end-ofsentence performance and therefore we were unable to detect any differences in on line processing of the two quantified phrases.

Turning towards the results for PWA, the main finding was that both the agrammatic and the non-agrammatic patients were able to arrive at both scope interpretations. This clearly suggests that PWA are capable of building complex syntactic representations, such as those required in assigning inverse scope interpretation to ambiguous doubly quantified sentences. Given that the dependencies involved in those representations are unpredictable, the data support the conclusion that sentence comprehension in aphasia is intact when the establishment of intrasentential dependencies do not require syntactic predictions, which is in line with our experimental hypothesis. This finding is also in line with the only previously published study investigating processing of scope ambiguities in agrammatism (see Saddy, 1995). Saddy's main observation was that the agrammatic patient who participated in the study did not exhibit difficulty deriving either of the possible interpretations for ambiguous sentences. Interestingly, PWA were more likely to endorse an inverse scope interpretation than a surface scope one. This performance pattern is in line with the overall performance pattern observed in this study. That is, overall participants (both PWA and controls) performed better in trials associated with inverse scope interpretations. It also expands some of Clark and Kar study's findings (2011). Using a self-paced reading task with picture verification, Clark and Kar showed that the items more likely to elicit errors were those associated with surface scope interpretations for a...every sentences, like those used in our experiment. They also found that the same items were more likely to elicit longer reaction times, a pattern that was not observed in our study.

Looking at the reaction time data, overall participants took longer to respond to trials that required them to assign inverse scope interpretations. Given that PWA performed more accurately in these trials (compared to trials in which they had to assign surface scope interpretations), we argue that this finding suggests that inverse scope interpretations are indeed more processing demanding than surface scope interpretations. This manifests as slower responses. This is only a hypothesis. The paradigm used in this experiment measures end-of-sentence responses, no inferences about real-time processing can be made. This topic therefore needs further investigation. Finally, PWA were slower in the mismatching conditions. Accurate performance on mismatch items depends on the ability to maintain in memory the meaning of the target sentence along with the conflicting interpretation of the picture, so that the discrepancy between them can be noted. Given that PWA suffer from limited processing resources due to their brain damage, we suggest that storage and manipulation of the two competing representations exceeds patients' processing capacity resulting in lower performance (see also Cupples \& Inglis, 1993)

A final question we need to answer before moving to the next experiment is why PWA, unlike controls, did not exhibit scope preferences that would result in less 
accurate performance across conditions. Or, in other words, why controls overall performed worse than PWA. A possible explanation is that scope preferences are attenuated in aphasia due to patients' processing limitations. A similar approach has been suggested by Clark and Kar (2011) to account for the finding that patients with semantic dementia arrived at both scope interpretations with equal facility whereas controls exhibited scope biases. According to the hypothesis put forward by the authors, cognitively unimpaired individuals exhibit biases towards specific interpretations of ambiguous doubly quantified sentences based on subsequent logical or pragmatic reasoning. These biases are attenuated in semantic dementia due to brain damage. In a similar vein, we suggest that scope preferences require intact cognitive abilities and, given the brain damage, are attenuated in aphasia.

\section{Experiment 2}

The structures tested in Experiment 2 include sentences with object contrastive foci (CFi). 'Focus' is the information highlighted in a proposition. For example, in the answer to a wh-question, the constituent that corresponds to the wh-expression constitutes the focus. The rest of the sentence functions as its background; that is, the focus is highlighted with respect to this material. By this criterion, the Selfish Gene is the focus in (5a). This type of focus is known in the literature as new information focus (throughout the paper bold small capital fonts indicate foci).

a. Q: What did John read?

A: John read THE SELFISH GENE.

b. A: John read The Extended Phenotype.

B: (No, you're wrong.) THE SELFISH GENE he read.

(from Neeleman \& Vermeulen, 2012)

Example (5b) exemplifies the use of contrastive focus, the kind of focus we are concerned with in this paper. The distinction between the two types of focus is based on both semantic and syntactic differences (Kiss, 1998). Specifically, CF semantically is an operator that expresses negation of an alternative proposition (which differs from the asserted proposition only in the choice of the focus). A CF is therefore quantificational and licensed to undergo A'-movement (often to the left periphery of the clause), in some languages obligatory so. The relevant movement may be understood as a scope-marking movement; it establishes the scope of negation in the alternative proposition (Neeleman \& van de Koot, 2012; Neeleman \& Vermeulen, 2012). Thus (5b), where CF has moved to the left periphery, must be understood as asserting that there is at least one other relevant book that John did not read, namely The Extended Phenotype. New information focus, on the other hand, is merely the carrier of new information and does not assert the negation of an alternative proposition. It therefore lacks quantificational properties and does not license movement. 
The positioning of CFi in Greek is flexible; both preverbal and postverbal object foci can be interpreted contrastively (Gryllia, 2009; Haidou, 2012). In (6a) the object $\mathrm{CF}$ has been moved to the preverbal position, but (6b) is also a felicitous reply, despite the fact that the object $\mathrm{CF}$ has remained in situ.

$$
\begin{aligned}
& \text { a. TON ADRA pleni i gineka ohi to agori. } \\
& \text { the }_{\mathrm{ACC}} \text { man }_{\mathrm{ACC}} \text { wash }_{3 \mathrm{rd} \text { SING }} \text { the }_{\mathrm{NOM}} \text { woman }_{\mathrm{NOM}} \text { not the boy } \mathrm{ACC}_{\mathrm{AC}}
\end{aligned}
$$

'THE MAN the woman is washing, not the boy.'

$$
\begin{aligned}
& \text { b. i gineka pleni TON ADRA ohi to agori. } \\
& \text { the }_{\mathrm{NOM}} \text { woman }_{\mathrm{NOM}} \text { wash }_{3 \mathrm{rdSING}} \text { the } e_{\mathrm{ACC}} \text { man }_{\mathrm{ACC}} \text { not the boy } y_{\mathrm{ACC}}
\end{aligned}
$$

In this paper we assume, following Neeleman \& Van de Koot $(2012,2008)$ that A'movement of a contrastive object focus takes place in order to mark the material that is included in its scope. This material is known as the domain of contrast. When the contrastive focus remains in situ, as in (6b), the domain of contrast is not marked in the overt syntactic representation. However, since we are still able to interpret the object focus contrastively, we must assume that the contrastive focus is assigned its scope at Logical Form through Quantifier Raising, the same operation involved in the assignment of inverse scope interpretation to ambiguous doubly quantified sentences. In a nutshell, through the application of Quantifier Raising in Logical Form, the in situ contrastive focus is interpreted in a position higher than its surface position and hence takes (contrastive) scope in this derived position.

The flexibility in the position of the object CF in Greek provides an appropriate minimal pair to explore our hypothesis that PWA confront difficulties when processing syntactic dependencies in highly predictive contexts whereas their sentence processing is relatively well preserved when syntactic dependencies are unpredictable. Specifically, sentences with moved CF such as (6a) contain a predictable filler-gap dependency, namely the dependency between the moved constituent and its base-generated position. On the other hand, in sentences with in situ CF such as (6b) the dependency that is created in Logical Form is unpredictable.

\subsection{Methods}

\subsubsection{Participants}

Six out of the seven participants with aphasia from Experiment 1 (all but P4) participated in Experiment 2 as well. PWA had an average age of 54.67 years (SD = 7.84; range $42-62$ years) and an average of 13.33 years of education ( $\mathrm{SD}=1.97$; range: $11-16$ years). Nine healthy adults also participated as a control group. Their average age was 43.67 years ( $\mathrm{SD}=14.80$; range: $25-62$ years) and they had an average of 12.78 years of education ( $\mathrm{SD}=5.29$; range: $6-21$ years). All participants were matched on educational level (Kruskal-Wallis $\chi 2(2)=0.11$, $p$-value $=0.95$ ). Demographic information for the group of controls is presented in Table 2. 
Table 2 Demographic information for the group of control participants.

\begin{tabular}{cccc} 
ID & Sex & Age & Education \\
\hline $\mathrm{C} 01$ & $\mathrm{~F}$ & 27 & $18 \mathrm{yrs}$ \\
$\mathrm{C} 02$ & $\mathrm{~F}$ & 25 & $16 \mathrm{yrs}$ \\
$\mathrm{C} 03$ & $\mathrm{M}$ & 34 & $14 \mathrm{yrs}$ \\
$\mathrm{C} 04$ & $\mathrm{M}$ & 35 & $21 \mathrm{yrs}$ \\
$\mathrm{C} 05$ & $\mathrm{~F}$ & 59 & $12 \mathrm{yrs}$ \\
$\mathrm{C} 06$ & $\mathrm{M}$ & 60 & $8 \mathrm{yrs}$ \\
$\mathrm{C} 07$ & $\mathrm{~F}$ & 62 & $6 \mathrm{yrs}$ \\
$\mathrm{C} 08$ & $\mathrm{~F}$ & 53 & $6 \mathrm{yrs}$ \\
$\mathrm{C} 09$ & $\mathrm{M}$ & 38 & $14 \mathrm{yrs}$ \\
\hline
\end{tabular}

\subsubsection{Stimuli}

A picture selection task was designed to test the comprehension of sentences with object CFi. Materials consisted of spoken sentences presented simultaneously with pictures. Stimulus sentences were spoken by a female native speaker of Greek at a natural rate and recorded in a soundproof recording booth at UCL's Chandler House research laboratory. Any noise before the onset and after the offset of the sentences was removed using Audacity (http://audacity.sourceforge.net/). A set of 60 pictures was designed by modifying images from royalty free clip art available on the Internet. The size of the pictures was $480 \times 320$ pixels.

The experimental items consisted of 20 introductory SVO sentences pronounced with neutral intonation, such as the one presented in (7a), and 40 target sentences with object CFi. In half of the target sentences the contrastively focused object appeared in the preverbal position (i.e. moved CF), as in (7b), whereas in the other half it appeared in the postverbal position (i.e. in situ CF), as in (7c).

a. Se auti tin ikona ('in this picture')

$\begin{array}{lllll}\mathrm{i} & \text { gineka } & \text { pleni } & \text { to } & \text { agori. } \\ \text { the }_{\mathrm{NOM}} & \text { woman }_{\mathrm{NOM}} & \text { wash }_{3 \mathrm{rd} \text { SING }} & \text { the } & \\ \mathrm{ACC} & \text { boy }_{\mathrm{ACC}}\end{array}$

'The woman is washing the boy.'

Se mia apo aftes tis ikones omos ('in one of these two pictures though')

b. TON ADRA pleni i gineka ohi to agori.

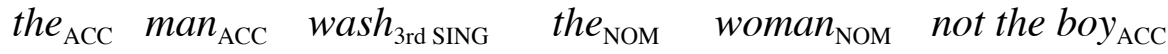

'THE MAN the woman is washing, not the boy.'

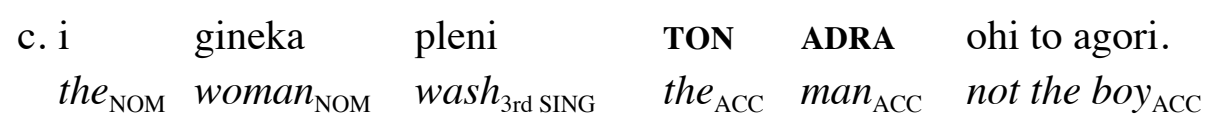

'The woman is washing THE MAN, not the boy.'

All sentences were semantically reversible. Introductory sentences were pronounced with a neutral, unmarked stress pattern. The target sentences were pronounced with the type of intonation that is typical for Greek speakers in marking contrastive focus. In the literature, there is no agreement as to whether contrastive stress should be 
described as a monotonal $\mathrm{H}^{*}$ (as in English) or a bitonal $\mathrm{L}+\mathrm{H}^{*}$ (see discussion in Georgakopoulos \& Skopeteas, 2010).

Apart from the experimental conditions, a filler condition consisted of 20 SVO sentences with CF in the subject position as in (8b) was also included. Finally, in order to assess PWA's comprehension abilities when processing syntactically simple sentences without any covert operations and contrastive intonation, we also included a baseline condition consisted of 20 simple SVO semantically reversible transitive sentences as in (8c). These sentences were produced with the type of intonation that is typical for Greek "all new" affirmative declarative sentences, where no word carries narrow focus. Both filler and baseline conditions included the same 20 introductory sentences as the experimental conditions. In total, there were 86 trials.

a. Se auti tin ikona ('in this picture')

$\begin{array}{lllll}\mathrm{i} & \text { gineka } & \text { pleni } & \text { to } & \text { agori. } \\ \text { the }_{\mathrm{NOM}} & \text { woman }_{\mathrm{NOM}} & \text { wash }_{3 \mathrm{rd} \text { SING }} & \text { the } & \\ \mathrm{ACC} & \text { boy }_{\mathrm{ACC}}\end{array}$

'The woman is washing the boy.'

Se mia apo aftes tis ikones omos ('in one of these two pictures though')

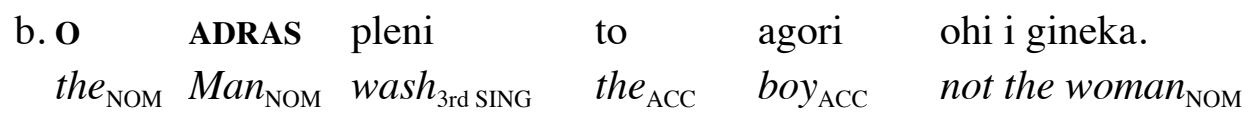

'THE MAN is washing the boy, not the woman.'

\begin{tabular}{|c|c|c|c|}
\hline $\begin{array}{l}\text { c. to } \\
\text { the } e_{\mathrm{NOM}}\end{array}$ & $\begin{array}{l}\text { koritsi } \\
\text { girl }_{\mathrm{NOM}}\end{array}$ & $\begin{array}{l}\text { agaliazi } \\
h u g_{3 \mathrm{rd} \text { SING }}\end{array}$ & $\begin{array}{l}\text { ton } \\
\text { the } \\
\mathrm{ACC}\end{array}$ \\
\hline
\end{tabular}

\subsubsection{Procedure}

After providing informed consent, participants were seated in a quiet testing room, in front of a computer. Stimuli were presented on a Dell laptop computer with a 15" screen using the SyntaxLab program developed for this study. First the template for the first part of the trials was presented and the instructions were given to participants. When ready, participants pressed the space bar with their dominant hand and the first trial began. Each trial consisted of two parts. In the first part, an introductory picture appeared in the upper half of the screen and an introductory sentence was heard via two speakers. The purpose of this part was to make sure that participants, especially patients, had understood the scene (i.e. who the participants in the event are, what the action being performed is, etc.), and to set up a context that would facilitate an interpretation of the target sentence as containing a CF (see left picture, Figure 7). When participants were ready, the second part of the trial began. For the PWA group, this was controlled by the experimenter by clicking the "next" button on the screen when patients nodded. For controls, the second part of the trial began automatically, few milliseconds after the end of the introductory sentence. In this part, two other pictures appeared in the lower half of the screen and the target sentence was heard twice. One picture correctly depicted the action represented by the target sentence; the 
other depicted the same action but with reversed thematic roles (see bottom pictures on right picture, Figure 7).

Participants were instructed to choose the picture that matched the target sentence presented in the second part of the trial by pressing one of the two response keys on the keyboard (i.e. $\mathrm{Z}$ for the left picture and $\mathrm{M}$ for the right picture). For half of the trials the matching picture appeared on the left, and for the other half on the right. Pictures were formatted to fill as much of the screen as possible. To make sure that participants would not respond before they had listened to the whole sentence, at the end of the spoken sentence a yellow square appeared on the screen, and participants were instructed to respond at this point. The pictures remained visible until a response was made. PWA were permitted to rest between trials as long as they wished. The procedure was slightly different for the control group. Participants performed the task in a quiet room using the same laptop. However, the experimenter was not present. Also, there was a five-second time-interval between the first and the second part of each trial and there were no breaks between the trials.

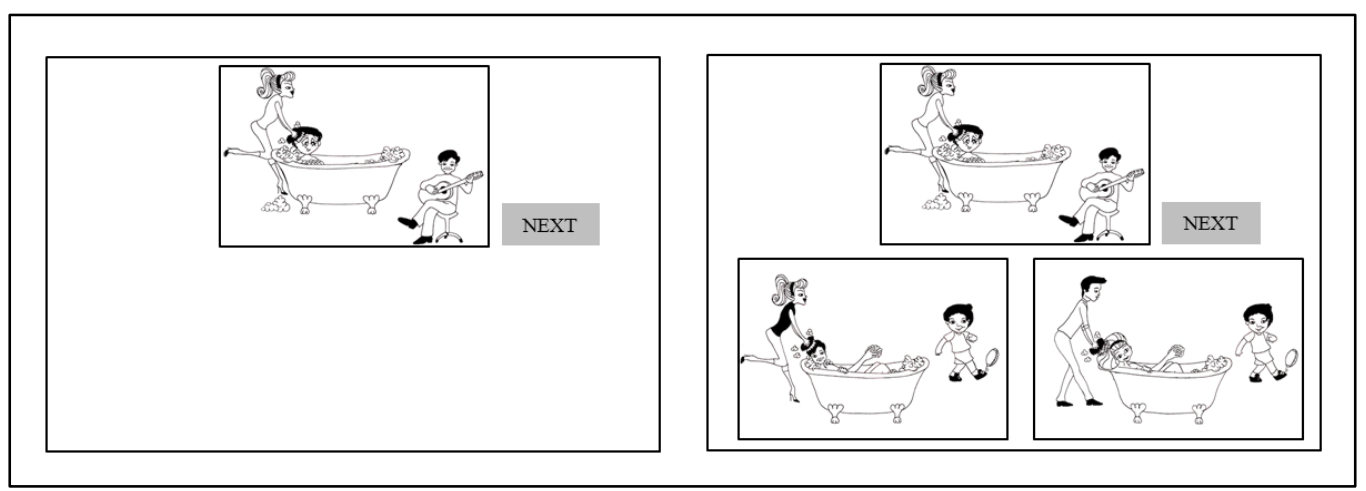

Figure 7 Left: sample of the first part of the trial. Participants are presented with an introductory/ background setting picture and an oral sentence (e.g. In this picture the woman is washing the boy). Right: Sample of the second part of the trial. Participants are orally presented with the target sentence either with moved contrastive focus (e.g. In one of these pictures, the MAN the woman is washing, not the boy) or with in situ contrastive focus (e.g. In one of these pictures, the woman is washing the MAN, not the boy), and another two pictures.

Reaction times and accuracy data were obtained from the offset of the spoken sentence to the key press. Responses before the offset of the spoken sentence were ignored. Participants were given six practice trials prior to the experiment and were permitted to repeat them as many times as they wished until they felt ready to undertake the experiment. Errors made on the practice trials were corrected. However, no feedback was provided during the actual experiment.

\subsubsection{Data analysis}

We used the same statistical analysis as in Experiment 1. The model was fitted with a random intercept for participants $(\mathrm{N}=15)$ and for concepts $(\mathrm{N}=20)$ and fixed effects for group (i.e. agrammatic patients, non-agrammatic patients and controls) and condition (i.e. moved $\mathrm{CF}$ and in situ CF). An interaction between the fixed factors was also added. Since controls were at ceiling there was a problem of complete separation and the logistic regression model would not fit. This problem was solved 
by randomly choosing two participants - one for the moved and another for the in situ contrastive conditions - and changing their response to incorrect. This affected in total only two out of 600 observations, i.e. $0.33 \%$ and meant the models could now fit. We also performed a post hoc analysis in which we compared participants' performance in the in situ CF condition with their performance when processing simple SVO semantically reversible sentences as in (8c). This comparison allowed us to explore whether PWA assign a contrastively focused interpretation in sentences with in situ CFi. Our hypothesis is that if they do and assuming that integration of prosodic information is resource demanding, then there should be differences in processing the two types of sentences especially for the group of agrammatic patients.

\subsection{Results}

\subsubsection{Accuracy}

Figure 8 presents the mean values for correct responses per group along with $95 \%$ confidence intervals. The statistical analysis revealed a main effect of group (LLR $\left.\chi^{2}(2)=21.06, p<0.001\right)$. Overall, the agrammatic patients were less accurate than controls (coefficient $=-5.63, \mathrm{SE}=1.17, \mathrm{z}=-4.82, \mathrm{p}<0.001$ ) and non-agrammatic patients (coefficient $=2.99, \mathrm{SE}=0.90, \mathrm{z}=3.33, \mathrm{p}<0.001$ ). On the other hand, the difference between the non-agrammatic patients and controls was not statistically significant (coefficient $=-2.65, \mathrm{SE}=1.44, \mathrm{z}=-1.84, \mathrm{p}=0.066$ ).

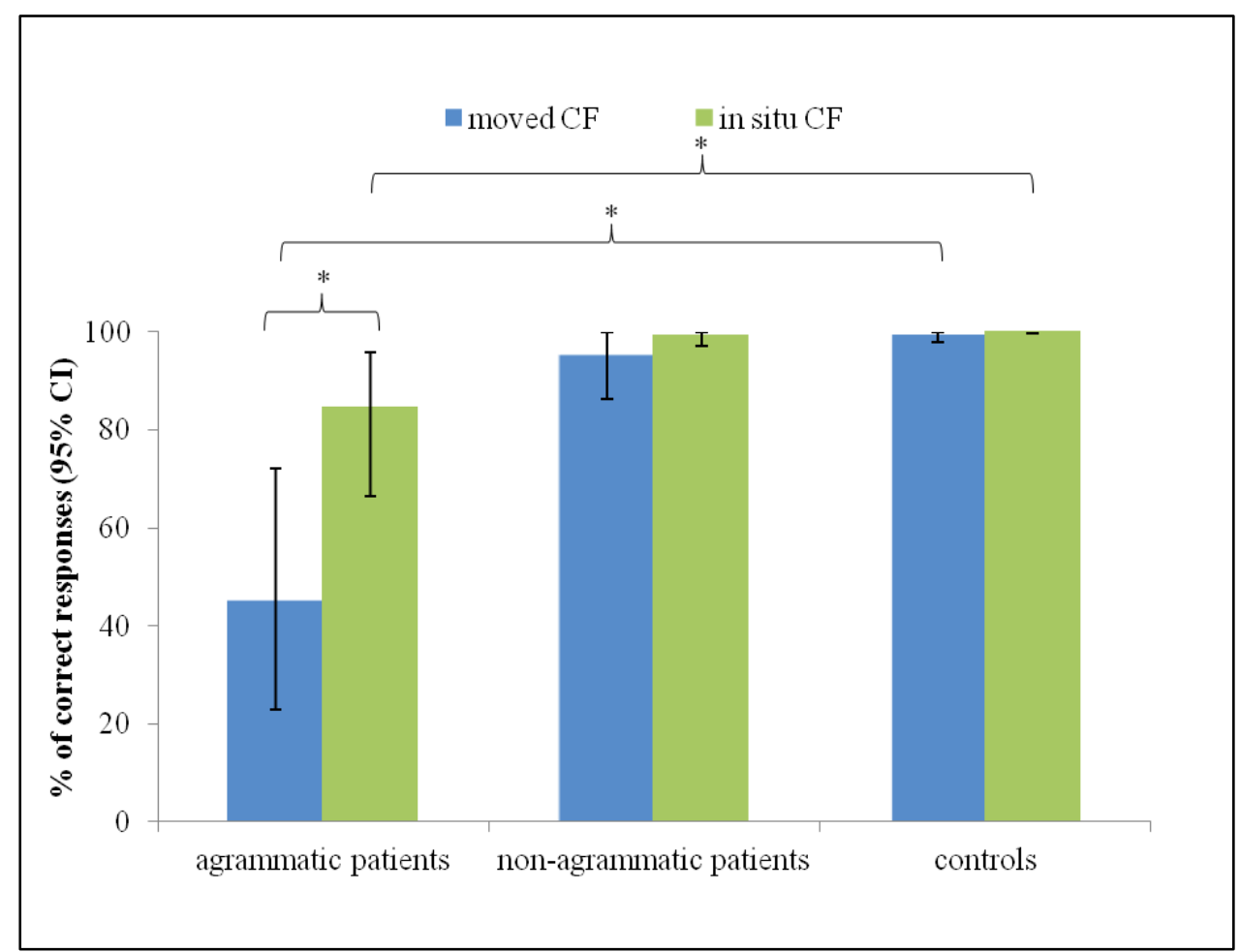

Figure 8 Comprehension accuracy as a function of condition and participants group, means (\%) and 95\% confidence intervals.

The main effect of condition was also significant (LLR $\left.\chi^{2}(1)=22.84, \mathrm{p}<0.001\right)$. Overall, participants were more likely to respond accurately when processing sentences with in-situ CF than sentences with moved $\mathrm{CF}$ (coefficient $=1.87, \mathrm{SE}=$ 
$0.42, \mathrm{z}=4.43, \mathrm{p}<0.001)$. The interaction between group and condition was not statistically significant $\left(\operatorname{LLR} \chi^{2}(2)=2.2, \mathrm{p}=0.34\right)$, nor were there main effects of age $\left(\operatorname{LLR} \chi^{2}(1)=0.35, \mathrm{p}=0.55\right)$ or education $\left(\operatorname{LLR} \chi^{2}(1)=1.34, \mathrm{p}=0.25\right)$.

Within group comparisons showed that the agrammatic patients performed significantly better on the in-situ CF condition compared to the moved CF condition (coefficient $=2.20, \mathrm{SE}=0.48, \mathrm{z}=4.6, \mathrm{p}<.0001$ ). They also performed significantly above chance in this condition ( $95 \%$ confidence interval above $50 \%$ ).

The comparison between sentences with in situ CF and SVO sentences revealed a main effect of group $\left(\operatorname{LLR} \chi^{2}(2)=11.14, p<0.01\right)$, similar to the one found in the main analysis. Also, the interaction between group and sentence type was also significant $\left(\operatorname{LLR} \chi^{2}(2)=7.76, \mathrm{p}<0.05\right)$. Individual comparisons showed that the difference in processing the two types of sentences was significantly larger in the agrammatic patients compared to controls $\left(\operatorname{LLR} \chi^{2}(1)=6.88, \mathrm{p}<0.01\right)$.

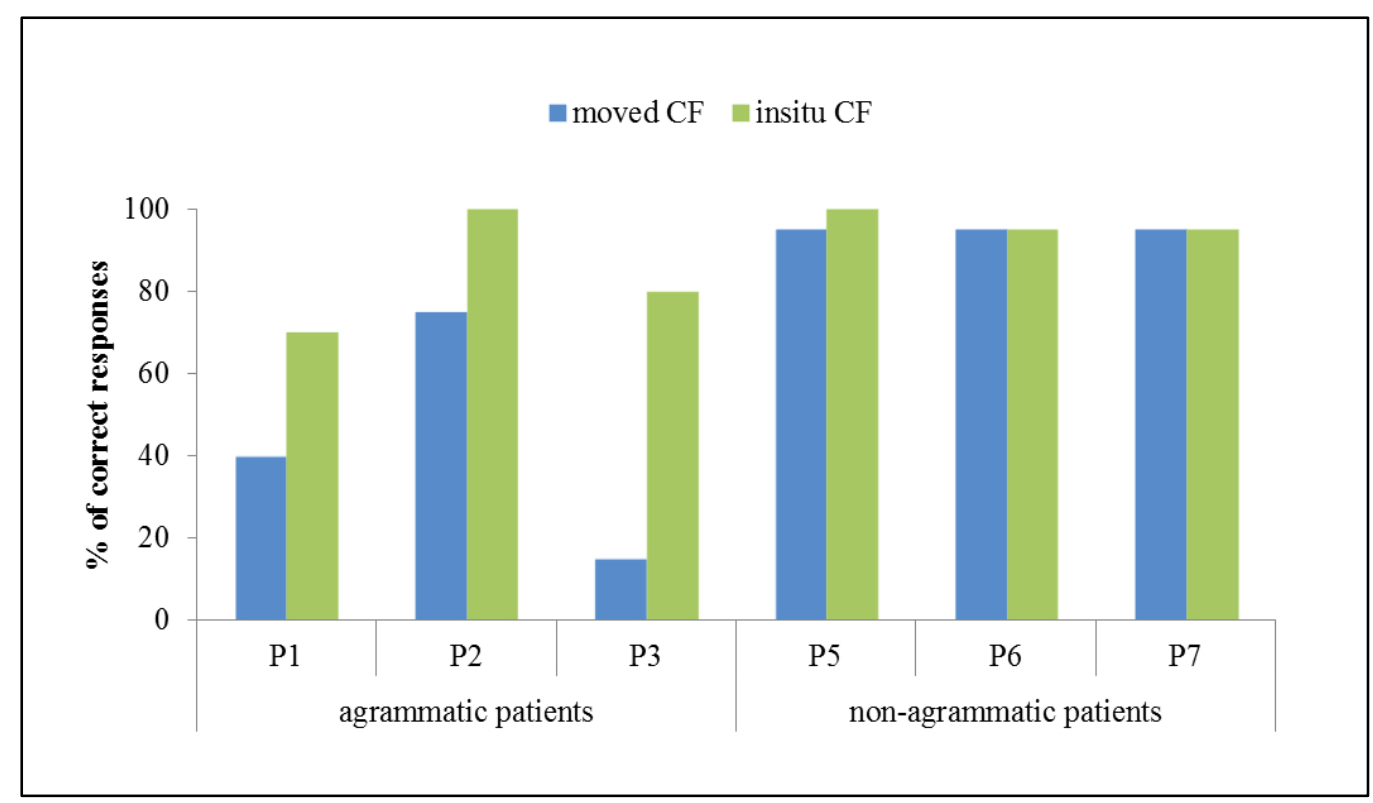

Figure 9 Individual comprehension accuracy means (\%).

Individual data inspection showed that there was variability within the group of agrammatic patients regarding comprehension of sentences with moved contrastive foci (see Figure 9). P3 exhibited very poor comprehension of these sentences, whereas P2 presented fewer difficulties compared to the other agrammatic patients. Interestingly, all agrammatic patients showed better comprehension of sentences with in situ CFi. Considering the non-agrammatic patients group, individual data revealed less variability; all non-agrammatic patients performed close to ceiling in both conditions. Overall, individual data seems to support the results of group analyses.

\subsubsection{Reaction times}

Overall, RTs of agrammatic patients were significantly longer than RTs of nonagrammatic patients $(95 \% \mathrm{CI} \in[0.04,1.43])$ and controls $(95 \% \mathrm{CI} \in[0.32,1.46])$ 
(see Figure 10). There was also an interaction between condition and group; agrammatic patients were slower in sentences with moved CFi compared to sentences with in situ $\mathrm{CFi}$, whereas the non-agrammatic patients and controls did not exhibit large differences between the two conditions $\left(\operatorname{LLR} \chi^{2}(2)=9.81, p=.007\right)$. There was no main effect of condition.

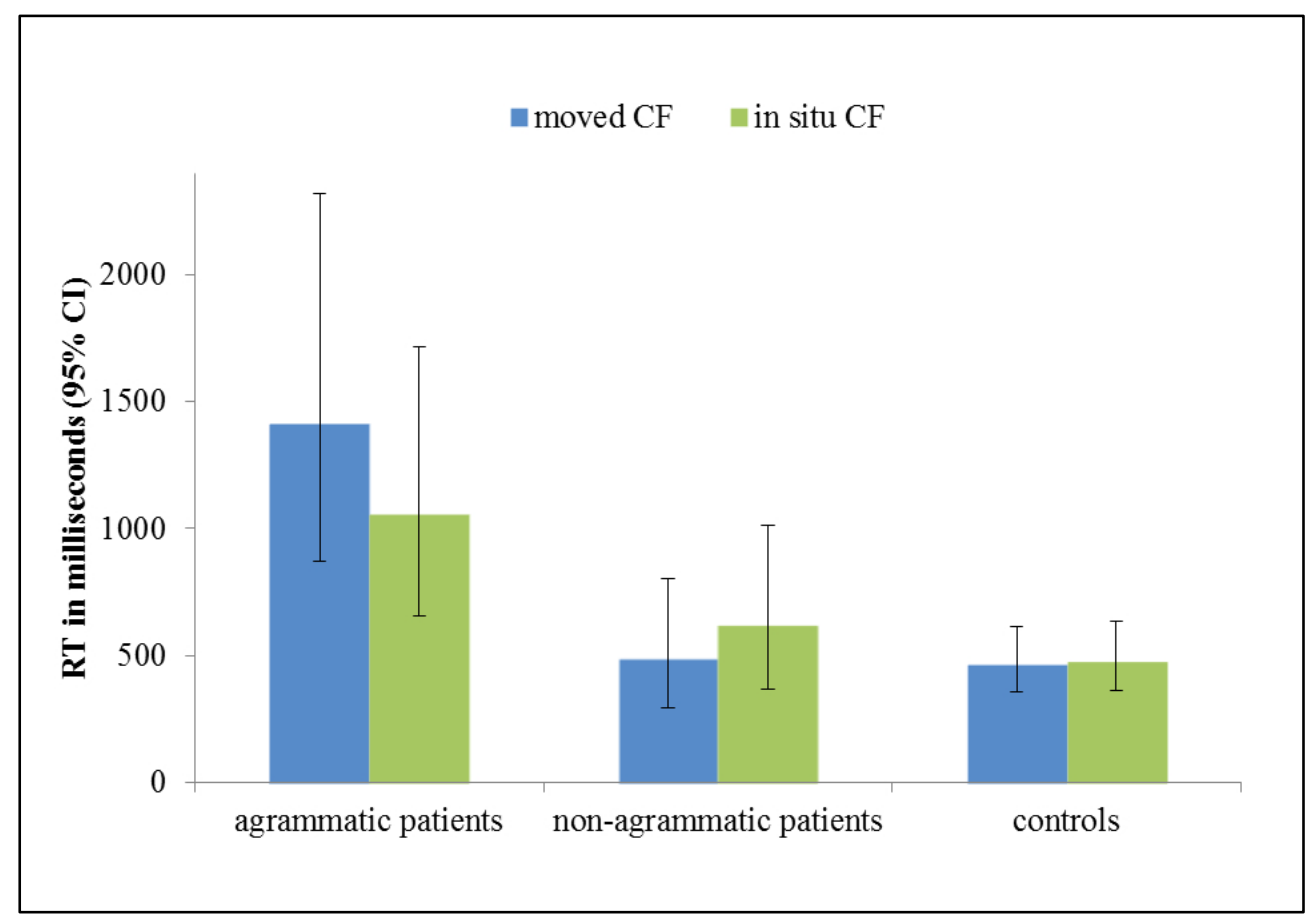

Figure 10 RTs in correct trials as a function of condition and participants group, milliseconds.

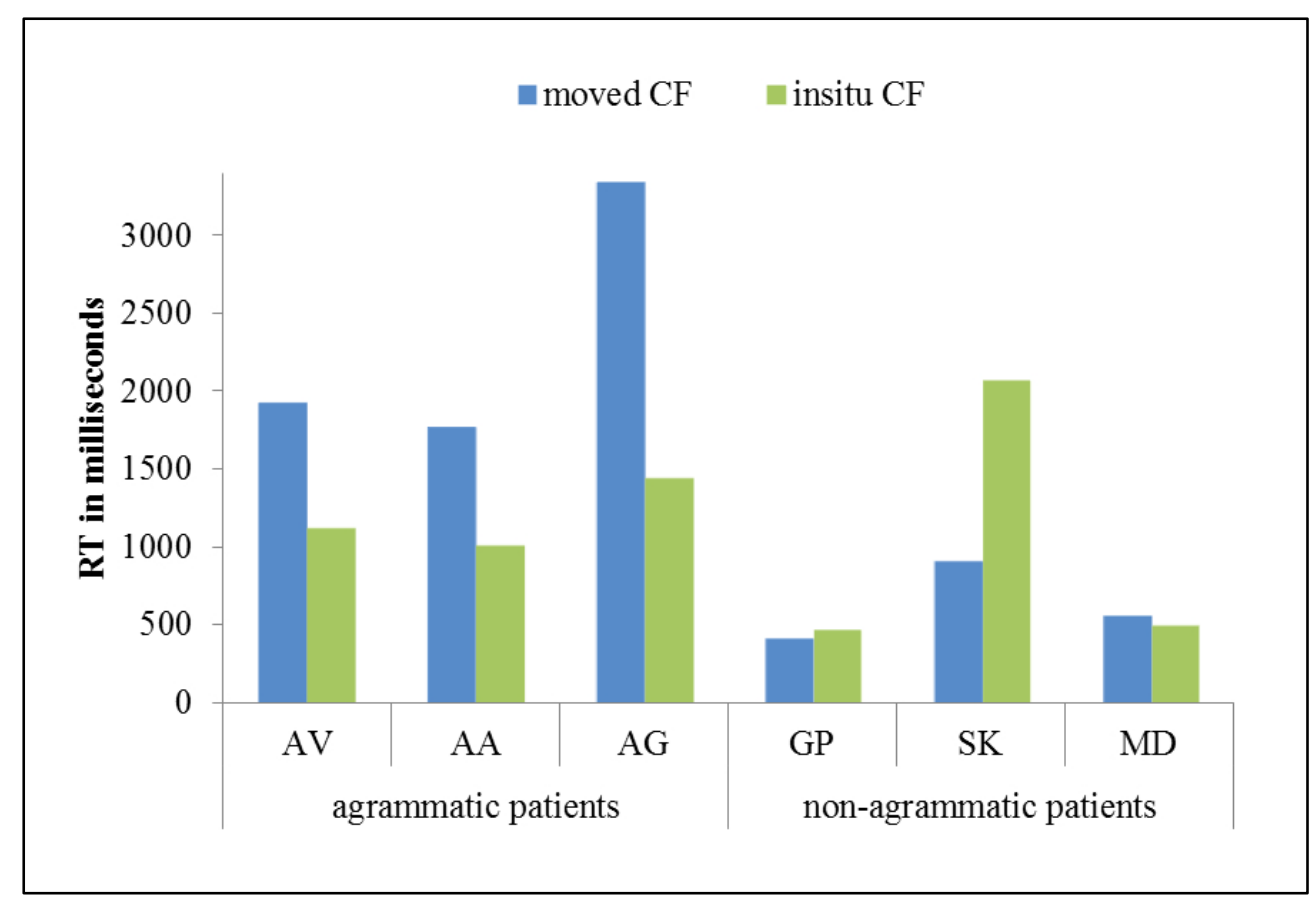

Figure 11 Individual RTs in correct trials, milliseconds.

Individual data analysis revealed that all agrammatic patients were faster in the in situ contrastive focus condition. The non-agrammatic patients were equally fast in 
both conditions, except P6 who was very slow in the in situ contrastive focus condition (see Figure 11).

\subsection{Discussion}

Experiment 2 examined comprehension of sentences with object $\mathrm{CFi}$. The reason we chose to test these structures is that CF displays quantificational properties and as such takes scope. Two types of such structures were tested, namely sentences with moved CFi and sentences with in situ CFi. In terms of syntactic predictions, the first type of sentences contains a predictable dependency, whereas the second type of sentences contains an unpredictable dependency. Investigating the two types of structure therefore allowed us to further explore our hypothesis that sentence comprehension deficits in aphasia may be related to the processing complexity introduced by syntactic predictions.

Controls performed at ceiling across the board; they were able to comprehend both types of sentences with equal facility and speed. This clearly suggests that in Greek both preverbal and postverbal object foci can receive a contrastive interpretation and unimpaired adults exhibit no preferences or biases towards one or the other structure. Therefore, we are confident to explain any differences observed in PWA's performance in terms of syntactic complexity.

As for PWA, two performance patterns were observed. The non-agrammatic patients performed well in both types of sentences and exhibited no difference in their RTs. This performance pattern is in line with their performance in the background sentence comprehension assessment. Specifically, the non-agrammatic patients did not exhibit any sentence comprehension difficulties when processing other types of movement-derived sentences (i.e. passives, relative clauses and $w$ h-questions) and therefore were expected to perform well in this experiment as well. The agrammatic patients, on the other hand, exhibited impaired comprehension of sentences with moved CF. This is in line with previous cross-linguistic findings suggesting that agrammatic patients confront difficulties when processing movement-derived sentences. This is also in line with their performance in the background sentence comprehension assessment. The fact that the agrammatic patients did not perform below chance indicates that they did not apply a linear agent-first strategy. Such a strategy would have resulted in below chance performance since movement of the contrastively focused constituent resulted in an OVS order (see Burchert et al., 2005; Hanne et al., 2015 for similar findings). The same patients, however, performed relatively better in the in situ CF condition. That is, although they performed worse than the non-agrammatic patients and controls, their performance in this condition was significantly better than their performance in the moved CF condition and well above chance. We argue that, taken together, these results suggest that sentence comprehension deficits in aphasia may be related to resource limitations and syntactic predictions, a factor that has been associated with increased complexity; comprehension of sentences whose interpretation requires the establishment of a predictable dependency, like sentences with moved CF, is impaired, because syntactic predictions are resource demanding and PWA have limited resources. On the other 
hand, comprehension of sentences whose interpretation requires the establishment of an unpredictable dependency, like sentences with in-situ CF, is relatively well preserved.

It could be argued that this difference in performance pattern is an artefact of the experimental design. Accurate performance in the in situ condition does not necessarily require the assignment of a contrastive interpretation to the object. In other words, it could be suggested that we cannot entirely rule out the possibility that the agrammatic patients processed sentences with in situ CF as simple transitive sentences with canonical word order and neutral intonation pattern and without performing any covert operation. There are two reasons to reject such a sceptical interpretation of our findings.

First of all, the contexts in which these sentences were processed strongly supported a contrastive interpretation of the in situ CFs. Since we can infer from PWA's performance in experiment 1 that they are able to assign scope to a quantified category through a covert operation, there is little reason to assume that they were unable to do so here.

Second, we performed a post hoc analysis in which we compared participants' performance in the in situ CF condition with their performance when processing transitive sentences with no contrastive intonation. This comparison revealed a significant interaction between group and sentence type; the agrammatic patients exhibited a bigger difference in processing the two types of sentences, with simple transitive sentences eliciting more accurate responses, whereas the other two groups did not exhibit differences. Clearly, something prevented agrammatic patients from responding as accurately in the in situ CF condition as when processing transitive sentences with no contrastive intonation. It seems reasonable that this "something" is their sensitivity to contrastive stress.

PWA's sensitivity to prosodic information has been the focus of previously published research in various languages. Gavarró and Salmons (2013) tested Catalan speaking PWA in a discrimination task, in which participants were orally presented with sentence pairs and asked to judge whether the sentences were identical or not. Among other pairs, the experimental items included contrastive focus/contrastive focus pairs and contrastive focus/neutral declarative pairs. Their main finding was that, although PWA were able to judge two sentences with contrastive stress as identical, they had difficulties in distinguishing between contrastively stressed sentences and neutral declarative sentences. However, based on their overall performance in other conditions as well, the authors concluded that PWA do not suffer from a prosodic disruption and that their perception of intonation is spared. In a study with German-speaking PWA Burchert and colleagues (2005) designed a truthvalue judgment task in which they tested comprehension of OVS sentences presented with either a neutral intonation or contrastive stress on the sentence initial object. The results showed that as a group PWA performed at chance level in both conditions. Individual data analysis revealed that only one patient improved his performance when the initial object was contrastively focused. The authors concluded that a contrastively focused constituent is not better understood than one belonging to an 
unmarked contour and therefore prosody does not enhance sentence comprehension in aphasia.

Taken together the results from the Catalan and German studies seem to suggest that PWA are not sensitive to contrastive stress and hence unable to use prosodic features as a cue for syntactic parsing (apart from the case of one participant reported in the Burchert et al. study). However, the appropriateness of the tasks used to tap processing of contrastive stress is questionable. While processing contrastively focused expressions, listeners identify those syllables that receive articulatory and tonal emphasis. However, accurate interpretation of $\mathrm{CF}$ requires computation of a contrast set typically presented in the discourse context. In these studies, participants were presented either with pairs of pictures and sentences (Burchert et al., 2005) or with pairs of sentences (Gavarró \& Salmons, 2013), but a context that could possibly allow for a contrastive interpretation was not provided. In other words, it may be the case that sentences with contrastive stress sounded unnatural out of context, which could have posed (additional) processing challenges to PWA.

Our hypothesis that the agrammatic patients did not perform at ceiling in the in situ CF condition due to the presence of contrastive intonation is in line with Avrutin and colleagues' suggestion that PWA are aware of the interpretative effect of contrastive stress. Specifically, in an early study Avrutin and colleagues (1999) investigated whether PWA are able to use contrastive stress as a determiner of reference shift for pronouns in coordinated structures like First, John hit Bill, and then MARY hit him and First, John hit Bill, and then Mary hit HIM, in which the prosodic nature of the pronoun is an important cue for the establishment of reference. PWA performed at chance level in both the unstressed and stressed condition. However, a significant disparity was found in their selection of the object NP as the referent; PWA chose the object NP as the referent of the pronoun $60.2 \%$ of the time in the unstressed condition but only $39.8 \%$ of the time in the stressed condition. On the basis of this disparity, the authors argued that the difficulties PWA exhibited in the interpretation of contrastively stressed pronouns in coordinated structures are not due to lack of knowledge of the interpretative effect of contrastive stress. Rather, the authors suggested that PWA have problems implementing this knowledge in the processing of complex structures, namely sentences with pronouns, which are independently known to pose problems in aphasia (for a similar study and results in Spanish see also Baauw, Ruigendijk, Cuetos, \& Avrutin, 2011).

\section{General Discussion}

The aim of this study was to explore the hypothesis that syntactic comprehension deficits in PWA may be related to resource limitations and a reduced ability to manage complexity in sentence processing. The experiments reported here investigate one factor that influences parsing complexity, namely the establishment of dependencies that require syntactic prediction. Independently motivated psycholinguistic theories, such as the Dependency Locality Theory, have suggested that generating and holding predictions about upcoming words and structures during sentence processing is associated with increased processing costs. Our hypothesis was 
that the processing cost associated with syntactic predictions might regularly exceed PWA's processing resources, which are limited due to the brain damage, resulting in comprehension failures. We dubbed this hypothesis the Complexity Threshold Hypothesis. Specifically, we anticipated that PWA's syntactic parser would suffer intermittent deficiencies when processing syntactic dependencies whose establishment requires syntactic predictions, whereas their sentence comprehension would be intact or relatively well preserved when no such predictions are required for the establishment of syntactic dependencies. To explore this hypothesis, we devised two experiments that investigate comprehension of scope relations by Greek-speaking agrammatic and non-agrammatic patients with aphasia. Two types of sentences were examined, namely ambiguous doubly quantified sentences (Experiment 1) and sentences with contrastive focus in the object position (Experiment 2). Our discussion here will focus on those results that are relevant to our experimental hypothesis and predictions.

The main finding of Experiment 1 was that the agrammatic patients that participated in our study, similar to the non-agrammatic patients and controls, were able to arrive at both scope interpretations, even the one that requires the establishment of an unpredictable dependency (i.e. inverse scope interpretation). This finding fits with our prediction that unpredictable dependencies are less resource demanding and therefore less likely to be associated with comprehension difficulties. This finding also indicates that PWA's sentence processing does not rely on nonsyntactic heuristics, as previous accounts of agrammatism have suggested. The use of heuristics would result in patients' low performance in the inverse scope condition, because the interpretation of the sentences included in this condition requires the universal quantifier every to take scope over the existential quantifier $a$. This results in processing of a marked argument order, in which the argument assigned the Theme thematic role (i.e. the object) appears before the argument assigned the Agent thematic role (i.e. the subject) in Logical Form. Alternatively, on the assumption that the agrammatic patients simply cannot compute the relevant Logical Form, their scope judgments should have aligned with the canonical surface position of the quantified arguments, always yielding an interpretation of surface scope. However, the results suggest that agrammatic patients apply heuristics only occasionally, when their syntactic parser fails. These failures are most likely to occur when processing sentences with increased processing cost and increased processing cost is plausibly associated with sentences that contain predictable dependencies. Another interesting finding of Experiment 1 was that overall PWA performed better than controls. We argued that this finding might be attributed to the fact that controls exhibited scope preferences towards specific interpretations. We also argued that scope preferences require intact cognitive abilities and that this is the reason why PWA did not exhibit such preferences.

Having established that agrammatic patients are capable of performing covert operations and processing unpredictable dependencies, and to find additional support for our hypothesis, we explored in Experiment 2 processing of sentences with moved CF and sentences with in situ CF. Data analysis revealed an interesting dissociation: 
the agrammatic patients exhibited impaired comprehension of sentences with moved CF but relatively well preserved comprehension of sentences with in situ CF. The non-agrammatic patients and controls performed equally well across conditions. This performance pattern is in line with the prediction outlined in the introductory section. We anticipated that sentences with moved CF would elicit more errors compared to sentences with in situ CF because the former type of sentences are more resource demanding due to the presence of predictable dependencies compared to the latter. We also found that the agrammatic patients did not perform at ceiling in processing sentences with in situ CF, despite the absence of syntactic predictions. We argued that this was due to the presence of contrastive intonation; the integration of prosodic information is resource demanding.

To summarize, this paper presented the findings of two interrelated experiments whose aim was to explore the processing of predictable and unpredictable dependencies in aphasia. The results indicate that sentence comprehension deficits in agrammatism are not due to loss of syntactic knowledge and use of heuristic strategies. Rather, in line with the Complexity Threshold Hypothesis, both experiments showed that these deficits may be related to PWA's limited resources for language processing and psycholinguistic factors that are known to affect the sentence comprehension of unimpaired speakers. The experimental manipulations allowed us to isolate one such factor, namely syntactic predictions.

The Complexity Threshold Hypothesis is in line with Mack and colleagues' (2013) suggestion that lexical-semantic predictions are slowed down in agrammatism. It is also in line with Hanne and colleagues' recent study (2015) exploring the use of morphological cues in processing OVS sentences. One of their main findings was that, although controls were able to integrate the morphological cue incrementally and override their SVO-template as quickly as after processing the first NP, their end-ofsentence reaction times were higher when processing OVS sentences compared to SVO sentences. The authors argued that this can be taken as an indication that predictive processes are resource demanding. As for PWA, they found that when processing OVS sentences the integration of the case-marking cue and the revision of the SVO-template was slowed-down, suggesting impaired predictive processes. In a similar vein, we argue that PWA are capable of generating syntactic predictions, but face resource limitations that may result in failure to construct or maintain the representations that support the prediction and creation of overt syntactic dependencies (that is, of gaps).

\subsection{The Complexity Threshold Hypothesis and other types of structures}

The Complexity Threshold Hypothesis could account for deficits in processing of other types of dependencies, not investigated in this study, that also require syntactic prediction, such as relative clauses, clefts and other A'-movement dependencies.

This study restricted itself to A'-dependencies, which naturally leads to questions concerning the effect of syntactic predictions on dependency types not investigated in this study and that could challenge our hypothesis, such as the Amovement dependency in passive sentences and sentences with unaccusative verbs 
(see Varkanitsa, 2015 for an extensive discussion of this issue). According to the standard analysis, the subject of these sentences originates postverbally and then moves to the left periphery. Therefore, these sentences contain a filler-gap dependency. This dependency, however, is unpredictable, in the sense that it becomes evident only after the listener/reader reaches the passive or the unaccusative verb. If this assessment of the empirical situation is correct, then our hypothesis would predict normal performance on these sentence types, contrary to fact. (Note that in a language like English though, the presence of the auxiliary be may provide an early cue for a passive structure.) There are, however, good reasons to suspect that the nature of the impairment in processing sentences with passive and unaccusative verbs is different from that in sentences with predictable dependencies such as object relatives, for example. In particular, it has been observed that in languages such as Spanish the comprehension of passives with postverbal subjects is also impaired in agrammatism, despite the fact that no overt movement takes place in these structures (Beretta, Harford, Patterson, \& Piñango, 1996; Beretta, Piñango, Patterson, \& Harford, 1999; Druks \& Marshall, 1995). Garraffa (2009) found that agrammatic patients performed below chance when judging the grammaticality of ungrammatical unaccusative sentences with postverbal subjects (see also Stavrakaki, Alexiadou, Kambanaros, Bostantjopoulou, \& Katsarou, 2011 for two agrammatic patients who were unable to produce such structures). Further evidence comes from a recent eye tracking study showing that impaired comprehension of passive sentences may be attributed to reduced automatic lexical activation and/ or lexical integration (Meyer et al., 2012).

The hypothesis that problems in the comprehension of sentences with passive and unaccusative verbs could be related not to the movement operation but to impaired lexical operations fits with a tradition in linguistic theory that takes the lexicon to be a 'module' of the grammatical system, with its own designated primitives and operations (see Siloni, 2003, and references cited there). On this view, comprehension of sentences with passive and unaccusative verbs relies on a lexical operation that transforms a transitive verb into a passive or unaccusative verb, respectively (Reinhart, 2002). Given that agrammatic patients have problems with these sentences also when their single argument remains in situ, it would be a reasonable hypothesis to link the problems witnessed in agrammatic comprehension and production with this lexical operation.

\subsection{Concluding remarks and future directions}

The experimental data presented in this paper indicate that agrammatic patients follow normal processing routines when processing sentences. However, their parser often fails due to their limited processing resources. One situation in which these failures are most likely to occur is during processing of syntactically complex sentences. What is innovative in our approach is that the account we propose for comprehension deficits in agrammatism is grounded in psycholinguistically motivated measures of syntactic complexity. Specifically, we suggest that comprehension failures are likely to occur when sentences require speakers to generate and maintain syntactic predictions in aid of gap creation, whereas in cases where no such predictions are 
required sentence processing is more successful. In other words, asyntactic comprehension in agrammatism may be attributed to patients' inability to generate or maintain syntactic predictions or, more general, to their inability to parse sentences whose complexity has exceeded a certain threshold due to their limited resources for language processing (i.e. Complexity Threshold Hypothesis).

We think this approach generates interesting research questions for future research; here we mention only a few. An important question concerns the role of other psycholinguistic factors in sentence processing in aphasia that we were unable to explore in this study, such as similarity-based interference and frequency. Further investigation of anaphora resolution in agrammatism (i.e. sentences with reflexives and pronouns) in relation to the Complexity Threshold Hypothesis is also needed. Another important question is how patients' deficits in generating and using syntactic predictions relate to working memory deficits. Finally, we believe that it would be of great interest to replicate the findings of this study using online measures, such as eye movements and self-paced listening times.

\section{Acknowledgment}

We would like to thank the individuals who participated in this study, especially the patients with aphasia. We would also like to thank Gordon Craig for an earlier version of statistical analysis of the data presented here. Special thanks also to Mike Coleman for creating the software used in the experiment. The first author received research funding from the Greek State Scholarship Foundation (IKY), the Alexander Onassis Public Benefit Foundation and the A.G. Leventis Foundation. The second author was supported by "IRAKLITOS II - University of Crete" of the Operational Program for Education and Lifelong Learning 2007-2013 of the NSRF (2007-2013), co-funded by the European Union (European Social Fund) and National Resources.

\section{References}

Altmann, G. T. M., \& Kamide, Y. (1999). Incremental interpretation at verbs: restricting the domain of subsequent reference. Cognition, 73(3), 247-64.

Altmann, G. T. M., \& Kamide, Y. (2007). The real-time mediation of visual attention by language and world knowledge: Linking anticipatory (and other) eye movements to linguistic processing. Journal of Memory and Language, 57(4), $502-518$.

Anderson, C. (2004). The structure and real-time comprehension of quantifier scope ambiguity. Northwestern University.

Avrutin, S. (2006). Weak Syntax. In Y. Grodzinsky \& K. Amunds (Eds.), Broca's Region (pp. 49-62). New York: Oxford University Press.

Avrutin, S., Lubarsky, S., \& Greene, J. (1999). Comprehension of contrastive stress by Broca's aphasics. Brain and Language, 70(2), 163-186. http://doi.org/10.1006/brln.1999.2154

Baauw, S., Ruigendijk, E., Cuetos, F., \& Avrutin, S. (2011). The interpretation of stressed and non-stressed pronouns in Spanish language breakdown. Aphasiology, 25(3), 386-408. http://doi.org/10.1080/02687038.2010.493295

Bartek, B., Lewis, R. L., Vasishth, S., \& Smith, M. R. (2011). In search of on-line locality effects in sentence comprehension. Journal of Experimental Psychology. 
Learning, Memory, and Cognition, 37(5), 1178-98.

Bates, D., Maechler, M., Bolker, B., \& Walker, S. (2013). lme4: Linear mixed-effects models using Eigen and S4. R package version 1.0-5. http://cran.Rproject.org/package $=1 m e 4$.

Beretta, A., Harford, C., Patterson, J., \& Piñango, M. (1996). The derivation of postverbal subjects: Evidence from agrammatic aphasia. Natural Language and Linguistic Theory, 14(4), 725-748.

Beretta, A., Piñango, M., Patterson, J., \& Harford, C. (1999). Recruiting comparative crosslinguistic evidence to address competing accounts of agrammatic aphasia. Brain and Language, 67(3), 149-68. http://doi.org/10.1006/brln.1999.2051

Burchert, F., Weidlich, C., \& Bleser, R. De. (2005). Focus in the left periphery: A cue to agrammatic sentence comprehension? Brain and Language, 95(1), 115-116.

Burkhardt, P., Piñango, M. M., \& Wong, K. (2003). The role of the anterior left hemisphere in real-time sentence comprehension: Evidence from split intransitivity. Brain and Language, 86(1), 9-22. http://doi.org/10.1016/S0093934X(02)00526-6

Caplan, D., Waters, G., DeDe, G., Michaud, J., \& Reddy, A. (2007). A study of syntactic processing in aphasia I: Behavioral (psycholinguistic) aspects. Brain and Language, 101(2), 103-150.

Clark, D. G., \& Kar, J. (2011). Bias of quantifier scope interpretation is attenuated in normal aging and semantic dementia. Journal of Neurolinguistics, 24(4), 401419. http://doi.org/http://dx.doi.org/10.1016/j.jneuroling.2011.01.002

Cohen, J., \& Cohen, P. (1983). Applied multiple regression/correlation analysis for the behavioural sciences (Lawrence E). Hillsdale, NJ.

Cupples, L., \& Inglis, A. L. (1993). When task demands induce "asyntactic" comprehension: A study of sentence interpretation in aphasia. Cognitive Neuropsychology, 10(3), 201-234. http://doi.org/10.1080/02643299308253461

Dickey, M. W., Choy, J. J., \& Thompson, C. K. (2007). Real-time comprehension of wh- movement in aphasia: Evidence from eyetracking while listening. Brain and Language, 100(1), 1-22.

Dickey, M. W., \& Thompson, C. K. (2009). Automatic processing of wh- and NPmovement in agrammatic aphasia: Evidence from eyetracking. Journal of Neurolinguistics, 22(6), 563-583. http://doi.org/10.1016/j.jneuroling.2009.06.004

Druks, J., \& Marshall, J. C. (1995). When passives are easier than actives: two case studies of aphasic comprehension. Cognition, 55(3), 311-331.

Dwivedi, V. D., Phillips, N. A., Einagel, S., \& Baum, S. R. (2010). The neural underpinnings of semantic ambiguity and anaphora. Brain Research, 1311(0), 93-109. http://doi.org/http://dx.doi.org/10.1016/j.brainres.2009.09.102

Ferrill, M., Love, T., Walenski, M., \& Shapiro, L. P. (2012). The time-course of lexical activation during sentence comprehension in people with aphasia. American Journal of Speech-Language Pathology / American Speech-LanguageHearing Association, 21(2), S179-89. http://doi.org/10.1044/10580360(2012/11-0109)

Fodor, J. (1982). The Mental Representation of Quantifiers. In S. Peters \& E. Saarinen (Eds.), Processes, Beliefs, and Questions SE - 5 (Vol. 16, pp. 129-164). Springer Netherlands. http://doi.org/10.1007/978-94-015-7668-0_5

Ford, M. (1983). A method for obtaining measures of local parsing complexity throughout sentences. Journal of Verbal Learning and Verbal Behavior, 22(2), 203-218. http://doi.org/10.1016/S0022-5371(83)90156-1 
Friederici, A. D., \& Kilborn, K. (1989). Temporal Constraints on Language Processing: Syntactic Priming in Broca's Aphasia. Journal of Cognitive Neuroscience, 1(3), 262-272. http://doi.org/10.1162/jocn.1989.1.3.262

Friederici, A. D., Steinhauer, K., Mecklinger, A., \& Meyer, M. (1998). Working memory constraints on syntactic ambiguity resolution as revealed by electrical brain responses. Biological Psychology, 47(3), 193-221. http://doi.org/10.1016/S0301-0511(97)00033-1

Garraffa, M. (2009). Minimal structures in aphasia: A study on agreement and movement in a non-fluent aphasic speaker. Lingua, 119(10), 1444-1457.

Gavarró, A., \& Salmons, I. (2013). The discrimination of intonational contours in Broca's aphasia. Clinical Linguistics \& Phonetics, 27(8), 632-646.

Gelman, A., \& Hill, J. (2007). Data analysis using regression and multilevel/hierarchical models (Cambridge ). Cambridge.

Georgakopoulos, T., \& Skopeteas, S. (2010). Projective vs. interpretational properties of nuclear accents and the phonology of contrastive focus in Greek. The Linguistic Review. http://doi.org/10.1515/tlir.2010.012

Gibson, E. (1998). Linguistic complexity: locality of syntactic dependencies. Cognition, 68(1), 1-76.

Gibson, E. (2000). The dependency locality theory: A distance-based theory of linguistic complexity. In Y. Miyashita, A. Marantz, \& W. O’Neil (Eds.), Image, language, brain (pp. 95-126). Cambridge, MA: MIT Press.

Goodglass, H., \& Kaplan, E. (1972). The assessment of aphasia and related disorders. Philadelphia, PA: Lea \& Febiger.

Gordon, P. C., Hendrick, R., \& Johnson, M. (2001). Memory interference during language processing. Journal of Experimental Psychology. Learning, Memory, and Cognition, 27(6), 1411-23.

Grodner, D., \& Gibson, E. (2005a). Consequences of the serial nature of linguistic input for sentenial complexity. Cognitive Science, 29(2), 261-90.

Grodner, D., \& Gibson, E. (2005b). Consequences of the serial nature of linguistic input for sentenial complexity. Cognitive Science, 29(2), 261-90. http://doi.org/10.1207/s15516709 $\operatorname{cog} 00007$

Grodzinsky, Y. (1995). Trace deletion, theta-roles, and cognitive strategies. Brain and Language, 51(3), 469-97. http://doi.org/10.1006/brln.1995.1072

Gryllia, S. (2009). Greek marks topics instead of foci. The Linguistic Review, 26(2-3), $177-205$.

Haarmann, H. J., \& Kolk, H. H. J. (1991). Syntactic priming in broca's aphasics: Evidence for slow activation. Aphasiology, 5(3), 247-263. http://doi.org/10.1080/02687039108248527

Haidou, K. (2012). The Syntax Pragmatics Interface of Focus Phenomena in Greek. School of Oriental and Afrikan Studies, University of London.

Hanne, S., Burchert, F., De Bleser, R., \& Vasishth, S. (2015). Sentence comprehension and morphological cues in aphasia: What eye-tracking reveals about integration and prediction. Journal of Neurolinguistics, 34, 83-111. http://doi.org/10.1016/j.jneuroling.2014.12.003

Hanne, S., Sekerina, I. A., Vasishth, S., Burchert, F., \& De Bleser, R. (2011). Chance in agrammatic sentence comprehension: What does it really mean? Evidence from eye movements of German agrammatic aphasic patients. Aphasiology, 25(2), 221-244. http://doi.org/10.1080/02687038.2010.489256

Ioup, G. (1975). Some universals for quantifier scope. In J. Kimball (Ed.), Syntax and Semantic (Vol.4) (pp. 37-58). New York: Academic Press. 
Johnson-Laird, P. N. (1969). On understanding logically complex sentences. Quarterly Journal of Experimental Psychology, 21(1), 1-13. http://doi.org/10.1080/14640746908400187

Kertesz, A. (1982). Western Aphasia Battery. New York: Grune and Stratton.

King, J., \& Just, M. A. (1991). Individual differences in syntactic processing: The role of working memory. Journal of Memory and Language, 30(5), 580-602. http://doi.org/10.1016/0749-596X(91)90027-H

Kiss, É. K. (1998). Identificational focus versus information focus. Language, 74, $245-273$.

Kolk, H. (1995). A Time-Based Approach to Agrammatic Production. Brain and Language, 50(3), 282-303.

Kurtzman, H. S., \& MacDonald, M. C. (1993). Resolution of quantifier scope ambiguities. Cognition, 48(3), 243-279. http://doi.org/http://dx.doi.org/10.1016/0010-0277(93)90042-T

Levy, R. (2008). Expectation-based syntactic comprehension. Cognition, 106, 11261177. Retrieved from http://philpapers.org/rec/LEVESC

Levy, R., Fedorenko, E., \& Gibson, E. (2013). The syntactic complexity of Russian relative clauses. Journal of Memory and Language, 69(4), 461-496.

Lewis, R. L., \& Vasishth, S. (2005). An activation-based model of sentence processing as skilled memory retrieval. Cognitive Science, 29(3), 375-419.

Lewis, R. L., Vasishth, S., \& Van Dyke, J. A. (2006). Computational principles of working memory in sentence comprehension. Trends in Cognitive Sciences, 10(10), 447-454. http://doi.org/http://dx.doi.org/10.1016/j.tics.2006.08.007

Love, T., Swinney, D., \& Zurif, E. (2001). Aphasia and the time-course of processing long distance dependencies. Brain and Language, 79, 169-170.

Mack, J. E., Ji, W., \& Thompson, C. K. (2013). Effects of verb meaning on lexical integration in agrammatic aphasia: Evidence from eyetracking. Journal of Neurolinguistics, 26(6), 619-636.

Mak, W. M., Vonk, W., \& Schriefers, H. (2002). The Influence of Animacy on Relative Clause Processing. Journal of Memory and Language, 47(1), 50-68. http://doi.org/10.1006/jmla.2001.2837

Mak, W. M., Vonk, W., \& Schriefers, H. (2006). Animacy in processing relative clauses: The hikers that rocks crush. Journal of Memory and Language, 54(4), 466-490. http://doi.org/10.1016/j.jml.2006.01.001

May, R. C. (1977). The grammar of quantification. MIT.

Meyer, A. M., Mack, J. E., \& Thompson, C. K. (2012). Tracking Passive Sentence Comprehension in Agrammatic Aphasia. Journal of Neurolinguistics, 25(1), 3143.

Neeleman, A., \& Van de Koot, H. (2008). Dutch scrambling and the nature of discourse templates. The Journal of Comparative Germanic Linguistics, 11(2), 137-189.

Neeleman, A., \& van de Koot, H. (2012). Towards a Unified Encoding of Contrast and Scope. In A. Neeleman \& R. Vermeulen (Eds.), The Syntax of Topic, Focus and Contrast: An Interface-Based Approach (pp. 39-76). Mouton de Gruyter.

Neeleman, A., \& Vermeulen, R. (2012). The syntactic expression of information structure. In A. Neeleman \& R. Vermeulen (Eds.), The Syntax of Topic, Focus and Contrast: An Interface-Based Approach (pp. 1-38). Berlin: Mouton de Gruyter.

Piñango, M. M. (2002). Cortical Reflections of Two Pronominal Relations. In H. J. Simon \& H. Wiese (Eds.), Pronouns: representation and processes (pp. 233- 
252). Berlin: John Benjamins Publishing.

R Core Team. (2013). R: A language and environment for statistical computing. R Foundation for Statistical Computing, Vienna, Austria. URL http://www.Rproject.org/.

Raffray, C. N., \& Pickering, M. J. (2010). How Do People Construct Logical Form During Language Comprehension? Psychological Science, 21(8), 1090-1097. http://doi.org/10.1177/0956797610375446

Reinhart, T. (2002). The Theta System - an overview. Theoretical Linguistics, 28, 229. http://doi.org/10.1515/thli.28.3.229

Saddy, J. D. (1995). Variables and Events in the Syntax of Agrammatic Speech. Brain and Language, 50(2), 135-150. http://doi.org/http://dx.doi.org/10.1006/brln.1995.1043

Saffran, E. M., Berndt, R. S., \& Schwartz, M. F. (1989). The quantitative analysis of agrammatic production: Procedure and data. Brain and Language, 37(3), 440479. http://doi.org/http://dx.doi.org/10.1016/0093-934X(89)90030-8

Siloni, T. (2003). Active Lexicon. Theoretical Linguistics, 28(3), 383-400. http://doi.org/10.1515/thli.28.3.383

Stavrakaki, S., Alexiadou, A., Kambanaros, M., Bostantjopoulou, S., \& Katsarou, Z. (2011). The production and comprehension of verbs with alternating transitivity by patients with non-fluent aphasia. Aphasiology, 25(5), 642-668.

Thompson, C. K., \& Choy, J. J. (2009). Pronominal resolution and gap filling in agrammatic aphasia: evidence from eye movements. Journal of Psycholinguistic Research, 38(3), 255-83.

Thompson, C. K., Tait, M. E., Ballard, K. J., \& Fix, S. C. (1999). Agrammatic aphasic subjects' comprehension of subject and object extracted Wh questions. Brain and Language, 67(3), 169-87.

Traxler, M., Morris, R. K., \& Seely, R. E. (2002). Processing Subject and Object Relative Clauses: Evidence from Eye Movements. Journal of Memory and Language, 47(1), 69-90. http://doi.org/10.1006/jmla.2001.2836

Tsapkini, K., Vlahou, C. H., \& Potagas, C. (2009). Adaptation and validation of standardized aphasia tests in different languages: Lessons from the Boston Diagnostic Aphasia Examination - Short Form in Greek. Behavioural Neurology, 22(3), 111-119. http://doi.org/10.3233/ben-2009-0256

Tunstall, S. L. (1998). The interpretation of quantifiers: Semantics and processing. University of Massachusetts Amherst.

Varkanitsa, M. (2012). Quantitative and error analysis of connected speech: Evidence from Greek-speaking patients with aphasia and normal speakersitle. In G. Fragaki, A. Georgakopoulos, \& C. Themistocleous (Eds.), Current Trends in Greek Linguistics (pp. 313-338). Cambridge: Cambridge Scholars Publishing.

Varkanitsa, M. (2015). Processing of syntactic dependencies in agrammatism: The role of predictability. University College London.

Vasishth, S., \& Lewis, R. L. (2006). Argument-head distance and processing complexity: Explaining both locality and anti-locality effects. Language, 82(4), 767-794.

\section{APPENDIX}

Table 1 PWA's lesion information. 
P1 Chronic infarct part of the left insula and part of the external capsule and left basal ganglia (including part of lentiform nucleus). The infarct extends into part of the inferior frontal gyrus, the precentral gyrus, superior temporal gyrus and inferior parietal lobule with significant amount of gliosis.

P2 Chronic left middle cerebral artery (MCI) infarct. Anteriorly it involves the left frontal lobe including the inferior frontal gyrus, middle frontal gyrus and precentral gyrus, while it extends into the superior and middle temporal gyrus and posteriorly into the inferior parietal lobule. The insular cortex, left basal ganglia (including lentiform nucleus and caudate nucleus), internal capsule, external capsule and most part of the left thalamus and left deep periventricular white matter are also involved. No age-related white matter changes are noted.

P3 Chronic infarct involving the left superior and middle temporal gyrus and part of the inferiortemporal gyrus, with significant gliosis extending into the inferior parietal lobule.

P4 missing

P5 Chronic infarct involving the left inferior frontal gyrus and extending into the insula and part of the putamen. It also involves the superior temporal gyrus and a small part of the inferior parietal lobule.

P6 missing

P7 Chronic left infarct involving the left insular cortex, left basal ganglia (including left lentiform nucleus) and extending into the inferior frontal gyrus, superior temporal gyrus and part of the inferior parietal lobule.

Table 2 Percentages $(\%)$ of correct responses in syntactic comprehension tasks.

\begin{tabular}{|c|c|c|c|c|c|c|c|}
\hline Conditions & P1 & $\mathbf{P 2}$ & P3 & $\mathbf{P 4}$ & P5 & P6 & P7 \\
\hline $\begin{array}{l}\text { wh-questions } \\
\text { who-subject questions }\end{array}$ & 60 & 100 & 60 & 50 & 100 & 100 & 100 \\
\hline who-object questions & 60 & 80 & 80 & 50 & 100 & 100 & 100 \\
\hline which-NP-subject questions & 80 & 90 & 70 & 100 & 100 & 100 & 100 \\
\hline which-NP-object questions & 50 & 50 & 50 & 70 & 100 & 100 & 100 \\
\hline passives & 40 & - & 30 & 60 & 90 & 100 & 80 \\
\hline $\begin{array}{l}\text { relative clauses } \\
\text { subject-relative clauses }\end{array}$ & 10 & - & 100 & 80 & 90 & 100 & 90 \\
\hline object-relative clauses & 40 & - & 30 & 20 & 90 & 100 & 90 \\
\hline
\end{tabular}

